\title{
流动化学在药物合成中的最新进展
}

\author{
赵东波 \\ (拜耳技术工程(上海)有限公司 上海 201507)
}

\begin{abstract}
摘要 作为不断成熟的一项过程强化技术, 流动化学近十年来不论在学术研究还是工业应用方面均取得令人瞩目的发 展; 综述了流动化学技术在药物合成中的最新研发进展. 当前的流动化学技术与其早期的研究相比有许多突破, 例如 不再局限于某类反应可行性概念的验证、越来越多的知名国际制药企业公开发展该技术以及已经有不少相关多步全合 成成功案例的报道等. 在简单介绍流动化学的优势、存在的挑战和应对之策之后, 列举大量实例从不同工艺类型重点 介绍流动化学在多步复杂药物合成工艺中的最新进展.
\end{abstract}

关键词 流动化学; 微反应器; 微反应技术; 药物合成; 多步流动合成; 工艺类型

\section{Updated Applications of Flow Chemistry in Pharmaceutical Synthesis}

\author{
Zhao, Dongbo \\ (Bayer Technology and Engineering (Shanghai) Co. Ltd., Shanghai 201507)
}

\begin{abstract}
As a further-developing process intensification technology, flow chemistry has achieved a striking development either in academic or industrial area in the past decade. Updated applications of flow chemistry in pharmaceutical synthesis are overviewed. Compared with its early research and development (R\&D), current flow chemistry has already shown a lot of breakthroughs. For instance, no more limitations to the proof of feasibility concept for some certain reaction, public development of this technology by more and more distinguished international pharmaceutical companies and quite a few corresponding reports of successful examples about multistep total synthesis of active pharmaceutical ingredients (APIs). Different process categorizations are emphasized with many API syntheses to demonstrate the up-to-date progress of this topic, after a brief introduction of the advantages, existing challenges and the corresponding solutions for flow chemistry.
\end{abstract}

Keywords flow chemistry; microreactor; microreaction technology; API synthesis; multistep flow synthesis; process categorization

自从 20 世纪 90 年代初 Manz 等 ${ }^{[1 a]}$ 首次提出了微全 分析系统(miniaturized total analysis systems, $\mu$-TAS)的 概念以来, 微反应技术(Microreaction technology)不论在 学术研究还是工业应用方面均取得令人瞩目的成就 ${ }^{[2]}$. 尤其近十年来的发展更为突飞猛进，目前为止已经发表 了成百上千篇关于微反应技术研究和应用的文献，而且 在医药、农药、材料、食品及其它多个行业的研发和实 际生产过程中得到了越来越多的应用. 此外, 基于微反 应器的学术期刊 ${ }^{[b, 1 e, 1 f]}$ 和国际专题会议 ${ }^{[3]}$ 也为该领域的 交流和发展提供了很好的平台.

\section{1 流动化学发展概述}

\section{1 相关术语介绍}

“微全分析系统(Miniaturized total analysis systems,
$\mu$-TAS)” ${ }^{[1 a]}$ : 是用于化学(或生物)分析的实验室工艺全 序列的微型集成系统. 该概念首先由 Manz 等 ${ }^{\left[{ }^{[b]}\right.}$ 于 1990 年提出, 之后由于其功能进一步拓展至反应领域而催生 出 “芯片实验室(Lab-on-a-chip)”、微机电系统(Microelectromechanical System, i.e. MEMS) 以及目前更加泛指 的 “微反应器(Microreactor)” 和 “微流控(Microfluidics)” 等术语.

“微反应器(Microreactor)” ${ }^{[1 c]}$ ：即 “微通道反应器 (Microchannel reactor)”，是利用精密加工技术制造的特 征尺寸在 10 到 $300 \mu \mathrm{m}$ 之间的微型反应器. 一般而言, 微反应器是微混合器、微反应器(停留时间容器)、微换 热器和微控制(传感)器等微通道化工设备的通称, 通常 作为连续流反应器(Continuous flow reactor)来使用(相对 于间歇反应器，即 Batch reactor). 这里的 “微” 主要表

*E-mail: tony.zhao@bayer.com

Received August 3, 2012; revised September 26, 2012; published online October 8, 2012 
明工艺流体的通道在微米级别, 而不是特指微分析系统 的微型尺寸或适合处理的微量样品. 微反应技术近十年 来在化学合成领域取得突破性的进展, 其反应通道的尺 寸也由于工艺放大的要求而扩展至毫米级别, 因此学术 界开始使用更加确切的 “流动化学(Flow chemistry)” 术 语.

“流动化学(Flow chemistry)” [1d]: 或称为 “连续流化 学(Continuous flow chemistry)” [19], 是指通过泵输送物 料并以连续流动模式进行化学反应的技术. 尽管流动化 学在诸如石油化工行业的大宗化学品生产中是非常成 熟的技术, 但在药物合成等精细化工行业尤其是实验室 研发阶段, 该技术还是相对较新的概念. 因此无论在学 术界还是工业界, 这一提法将使连续流工艺的开发成为 关注的重点, 而不再局限于微通道反应器本身. 基于这 一概念, 目前已经有相关的学术期刊 ${ }^{[1 e, 1 f]}$ 和商品化的设 备发行或发售.

\section{2 流动化学与药物合成}

作为微反应技术与化学合成的交叉学科，不断发展 的流动化学(Flow chemistry)技术已经实现了从早期的 某些特定反应可行性概念的验证到多步连续复杂合成 的跨越. 尤其在药物合成方面 ${ }^{[4]}$, 越来越多的知名国际 制药企业改变过去严加保密的策略而开始公开发展该 技术，表 1 中列举了近年来各大制药公司利用流动化学 针对不同类型反应进行研发的进展情况.

\section{3 流动化学技术的优势}

相对于传统的间歇反应工艺，流动化学具有高速混 合、高效传热、窄的停留时间分布、重复性好、系统响 应迅速、便于自动化控制、几乎无放大效应、在线的化 学品量少以及安全性高等优势. 由于已经有大量文献和 综述 ${ }^{[17]}$ 进行了这方面的阐述，本文不再赘述. 据统计， 在有机合成和催化反应中大约有 $20 \% \sim 30 \%$ 的反应工

表 1 近年来各大制药公司利用流动化学的研发举例

Table 1 R\&D examples via flow chemistry in some international pharmaceutical companies in recent years

\begin{tabular}{|c|c|c|c|c|}
\hline 年份 & 公司名称 & 反应类型 & 参考文献 & 备注 \\
\hline $1998 \sim 2000$ & Merck AG (Germany) & Desymmetrization of thiourea etc. & [5] & \\
\hline $2000 \sim 2001$ & Sigma-aldrich & Hydrolysis of ester etc. & {$[6 \mathrm{a}, 6 \mathrm{~b}]$} & \\
\hline $2000 \sim 2001$ & SmithKline Beecham (UK) & Wittig reaction & {$[7 \mathrm{a}, 7 \mathrm{~b}]$} & \\
\hline $2001 \sim 2002,2004$ & Novartis & $\begin{array}{l}\text { Mukaiyama aldol reaction; Peptide } \\
\text { synthesis; Diastereoselective alkylation }\end{array}$ & {$[8 \mathrm{a} \sim 8 \mathrm{c}]$} & \\
\hline $2002 \sim 2003$ & GSK & $\begin{array}{l}\text { Domino (Hantzsch synthesis and aldol- } \\
\text { Diels-Alder reaction); Knorr synthesis } \\
\text { (pyrazoles); Enamine synthesis }\end{array}$ & {$[9 \mathrm{a}, 9 \mathrm{~b}]$} & \\
\hline 2004 & Johnson-Johnson & $\begin{array}{l}\text { Methyl carbamate synthesis; Newman- } \\
\text { Kuart rearrangement (carbamothioate); } \\
\text { Piperidone Ring-Expansion (ethyl } \\
\text { diazoacetate); Organolithium reagent }\end{array}$ & {$[22]$} & Tramadol \\
\hline 2005 & Siemens (US) & $\begin{array}{l}\text { Multistep synthesis of a radiolabeled } \\
\text { imaging probe }\end{array}$ & {$[20]$} & Radiolabeled Imaging Probe \\
\hline $2005 \sim 2006$ & Ube Industries (Japan) & Swern oxidation & {$[10 \mathrm{a}, 10 \mathrm{~b}]$} & \\
\hline 2006 & DSM & Ritter reaction & {$[11 \mathrm{a}, 11 \mathrm{~b}]$} & \\
\hline 2005,2008 & Brystol-Meyers-Squibb & $\begin{array}{l}\mathrm{Pd} / \mathrm{C} \text { catalyzed hydrogenation; } \\
\text { Gas-liquid oxidation }\end{array}$ & {$[45]$} & 6-Hydroxybuspirone \\
\hline $2006 \sim 2008$ & Lonza Ltd & Grignard and organolithium reagents & {$[12 \mathrm{a} \sim 12 \mathrm{c}]$} & \\
\hline $2007 \sim 2009$ & BHC-BSP & Ozonolysis and fluorination of steroids & {$[24 b]$} & Vitamin D precursor \\
\hline $2008 \sim 2010$ & Pfizer (UK) & $\begin{array}{l}\text { Curtius rearrangement; Nitration } \\
\text { reaction; Iododeamination; Sandmeyer } \\
\text { reaction; }[3+2] \text { dipolar cycloaddition }\end{array}$ & {$[13 \mathrm{a} \sim 13 \mathrm{c}]$} & \\
\hline $2009 \sim 2010$ & Eli Lilly and Company & $\begin{array}{l}\text { Biphasic azide alkylation; } \mathrm{O}_{2} \text {-mediated } \\
\text { oxidation of alcohols; Azide process }\end{array}$ & {$[14 \mathrm{a}, 14 \mathrm{~b}, 48]$} & NK1-II antagonists \\
\hline 2009,2012 & AstraZeneca & $\begin{array}{l}\text { Bromination reaction with DMF; } \\
\text { Organolithium reagents etc. }\end{array}$ & {$[15,23 b]$} & Inhibitor AZD6906 \\
\hline 2012 & Actelion Pharmaceuticals Ltd & Diels-Alder reaction at high temperature & {$[16]$} & \\
\hline
\end{tabular}


艺 $^{[18]}$ 可以通过流动技术在收率、选择性或安全性等方面 得到优化和提升.

\section{4 流动化学技术的机遇和挑战}

由于近年来制药工业对于新药开发的新要求, 药物 合成化学相应地需要进行以下调整: (1)更加快速地合成 目标分子; (2)同时要求选定合成路线可以实现快速再合 成以及方便随后的工艺放大; (3)可以为药物设计者扩展 可以利用的化学空间即多样化合成. 由于药物合成工艺 优化与时效之间的平衡导致长期以来绝大多数已有成 熟工艺均为间歇操作, 但是对于新药从发现阶段到生产 的全周期研究表明, 流动合成的潜在优势使其成为未来 药物合成的新策略和创新的重要源泉 ${ }^{[4]}$.

以 Pfizer 公司药物发现过程为例 ${ }^{[4 b]}$, 几乎每一阶段 (最初采样即 Initial hits、先导药物发现即 Lead discovery、 候选药物搜索即 Candidate seeking 以及候选药物预知即 Candidate alert notice)均可以通过流动化学进行改进或 提升. 流动化学技术在药物合成中的机遇可以总结如 下: (1)可以提升选择性和安全性差的间歇工艺; (2)方便 在线检测, 产品质量可控; (3)工艺设置和规模的灵活性 高, 方便工艺优化和放大; (4)可以与其它工艺技术如光 化学、微波化学(或传统批次工艺)和连续分离工艺有机 集成或联合使用; (5)处理好衔接技术后(如中间体预处 理、溶剂切换和试剂兼容性等问题), 可以实现连续多步 流动合成的复杂工艺 ${ }^{[17 d]}$.

诚然, 流动化学要想真正成为药物合成中的通用方 法所面临的挑战也是巨大的. 首先, 相对于大量文献中 发表的系统性间歇工艺过程, 流动化学工艺的开发利用 才刚起步因而所能提供参考的成功案例有限. 其次, 对 于流动和间歇工艺过程的诸如可操作性和经济性进行 评估, 目前看来流动化学并不是占有绝对优势 ${ }^{[19 a, 19 b]}$. 再次, 许多已知的间歇工艺要转换为流动工艺必须进行 比较大的调整, 例如有固体物料参与或产生的工艺过 程 ${ }^{[19 \mathrm{c} \sim 19 \mathrm{f}]}$ 和涉及高粘度物料或中间体的反应等. 最后, 有不少文献对于连续流动工艺的优势有些夸大, 作者认 为将来仍需要不断创新的理念和设备才能使流动化学 有进一步的突破.

\section{2 流动化学在药物合成中的案例分析}

前面提到, 当前的流动化学技术与其早期的研究相 比有许多突破, 例如不再局限于某类反应可行性概念的 验证、越来越多的知名国际制药企业公开发展该技术以 及已经有很多进行多步全合成成功案例的报道等. 在简 单介绍流动化学的优势、存在的挑战和应对之策之后, 本文希望通过大量的案例分析来介绍流动化学在药物 合成中的最新进展. 下面主要针对几类不同的工艺类型
进行系统阐述: (1)生物有机合成; (2)有机金属试剂参与 的连续合成; (3)气一液两相反应; (4)光反应连续合成; (5) 固载催化剂(柱)和固载试剂(柱)参与的连续合成; (6)微 波技术与连续流合成; (7)连续多步流动合成和(8)其它流 动合成. 由于很难找到统一的分类标准况且很多实例均 涉及多种反应类型，因此下文不少单一案例中可能同时 包含上述的某几类工艺类型.

\section{1 生物有机合成}

生物有机化学可以定义为一门研究所有涉及到生 物分子的化学过程的学科. 这个广阔的领域包括使用生 物催化剂来合成和篮选、在体外制备生物分子如 DNA 以及在一个生命体如细胞内进行有机反应. 微反应器技 术在该领域的发展和应用始于 20 世纪 90 年代开发的微 型全分析系统 $(\mu-\mathrm{TAS})^{[1 \mathrm{a}]}$, 在诊断检测方面取得了令人 瞩目的成就; 这其中包括酶连接的免疫吸附剂测定 (ELISAs) 以及最新开发的酶介导蛋白裂解技术和后续 的质谱分析技术(应用于蛋白质组). 目前微反应技术已 经进入生物催化领域; 相比以前, 酶更多应用于合成而 非诊断. 在微反应系统中进行生物催化背后的原因实际 上包含两个方面. 第一, 小型化的微反应器可以使酶的 用量很小, 特别当需要测定酶动力学过程的时候. 第二, 微反应器在合成中的经典优势, 即更好的控制传热、传 质过程以及更高比表面积, 使得利用生物催化剂开发优 化方法更加便捷, 特别是处理快速反应的时候. 此外, 最新的生物有机化学关注于研究细胞中的化学反应过 程. 使用微反应技术可以研究各种分子对单一细胞的行 为和对代谢过程的影响, 有理由相信它会帮助我们发展 更有效的药物笁选过程和生物技术. 生物有机化学在诊 断检测方面的应用以及针对细胞在芯片上近期的研发 不会在下文中讨论，由于篇幅有限仅介绍在生物探针和 生物催化方面天然产物合成的两个例子 ${ }^{[20,21]}$.

\subsection{1 放射性标记成像探针的多步合成}

位于美国 Culver 的西门子医疗解决方案美国公司 分子成像部门和学术界的几位合作者曾经利用集成的 微流体设备, 合成了一种 $\left[{ }^{18} \mathrm{~F}\right]$ 氟放射性标记的分子成像 探针即 2-脱氧-2- $\left[{ }^{18} \mathrm{~F}\right]$ 氟- $D$ - 葡萄糖(2-deoxy-2- $\left[{ }^{18} \mathrm{~F}\right]$ fluoro$D$-glucose)，该工作发表在 2005 年的 Science 杂志上 ${ }^{[20]}$. 如 Figure 1 所示, 该合成包括五步连续工艺, 分别为 $\left[{ }^{18} \mathrm{~F}\right]$ 氟的浓缩、水的蒸发、放射性氟化、溶剂交换以及 水解脱保护. 这是以流动方式进行自动多步合成最早的 例子之一, 将该多步化学反应合成转换为集成有微通道 和阀并能进行连续流传输和控制的微流设计方案请参 见原文 ${ }^{[20]}$.

与传统的自动合成相比, 利用微反应器工艺技术获 得产品的放射性化学产率和纯度更高而且合成时间更 


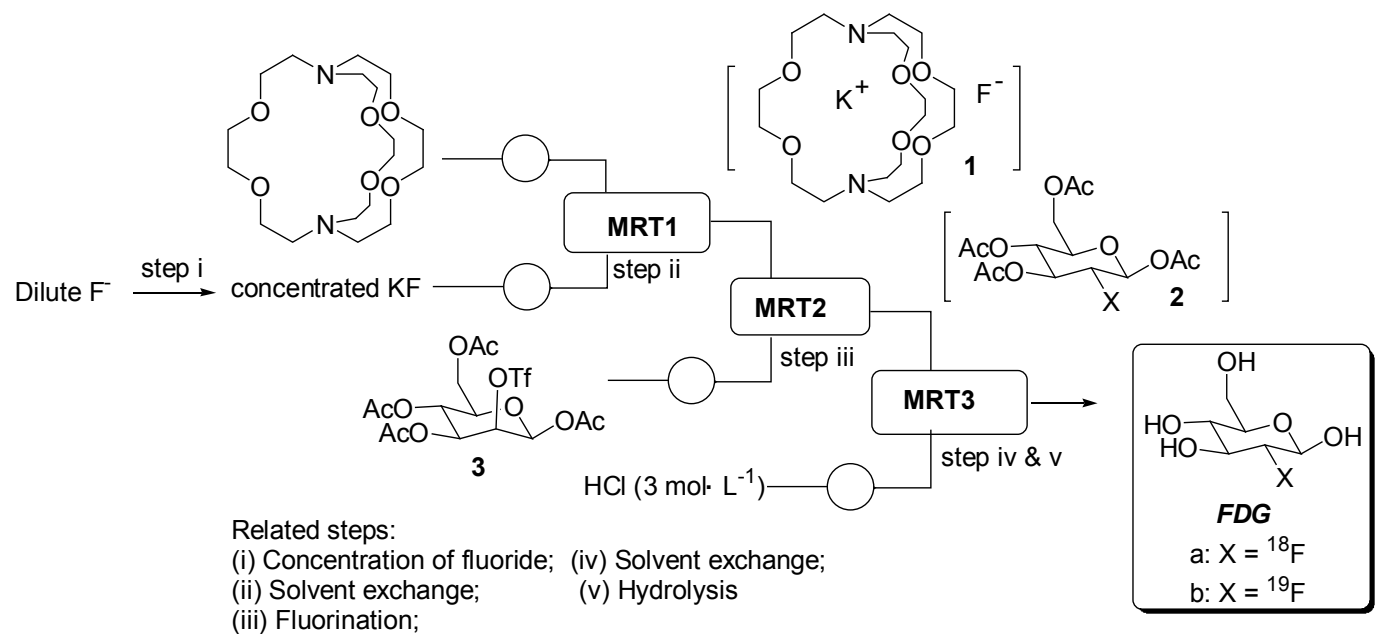

图 1 放射性标记成像探针的多步合成

Figure 1 Multistep synthesis of radiolabeled imaging probe

短. 由于需要针对小鼠上的正电子发射断层进行扫描成 像研究而且 $\left[{ }^{18} \mathrm{~F}\right]$ 氟的半衰期 $t_{1 / 2}$ 为 $110 \mathrm{~min}$, 因此对于不 同剂量的 2-脱氧-2-[ $\left.{ }^{18} \mathrm{~F}\right]$ 氟- $D$-葡萄糖进行快速合成是十 分重要的. 而目前通用的方法在大约 $50 \mathrm{~min}$ 的单次制备 中可以生产 $10 \sim 100$ 剂量的放射性标记化合物, 而且需 要使用昂贵的商业化合成仪.

\section{1 .2 大海米酰胺的合成}

除了传统有机合成之外, 微反应器还可以应用在酶 催化合成上. 生物催化反应中使用的酶必须在温和条件 下进行反应才具有效率和选择性. 由于微通道具有更高 的传质性能和高的比表面积等优点, 使得通过酶固定微 反应器在这一方面可以实现二者相关, 而且酶的使用可 以实现最优化. 相对而言, 小型化的生物催化合成微反 应器仅需很少量的催化剂. 新木脂素天然产物大海米酰 胺(grossamide)的合成就是一个很好的例子 [21].

如 Figure 2 所示, 英国剑桥大学的 Ley 小组 ${ }^{[21]}$ 首先 通过 PS-HOBt 固载试剂柱将底物酸和胺进行酰胺化得 到目标二聚体的单体 4. 该单体随后从 $\mathrm{PS}-\mathrm{SO}_{3} \mathrm{H}$ 净化柱
中被洗提出来, 并与另一股含有过氧化氢一尿素复合物 和磷酸二氢钠缓冲液的输入液以 $3: 1$ 的比例进行混合. 合并后的液流通过含有辣根过氧化物酶的第三根硅胶 柱进行氧化二聚反应，就得到了大海米酰胺. 虽然当前 是手动切换装置, 但通过一紫外探测器作为触发器, 使 用电脑就可以对系统实现进一步的自动化控制. 此外, 该论文对于开发多步合成而言许多重要技术和程序也 进行了经验总结和理论探讨，可以看作该领域里程碑式 的工作之一. 这些重要的技术和程序为未来多步合成研 发指明了方向，其中包括：(1)无论是起始原料的加入还 是产品的洗提，都可以利用液体处理器来控制; (2)在整 个初始优化程序中, 都可使用在线 HPLC 监测并迅速优 化每个反应步骤; 并且(3)该系统采用了不少固载柱和 阀门, 并通过在线紫外检测器监控流体的反应进程, 这 为将来实现全自动化控制做好准备.

\section{2 有机金属试剂参与的连续合成}

药物合成中也有不少是活泼有机金属化合物参与

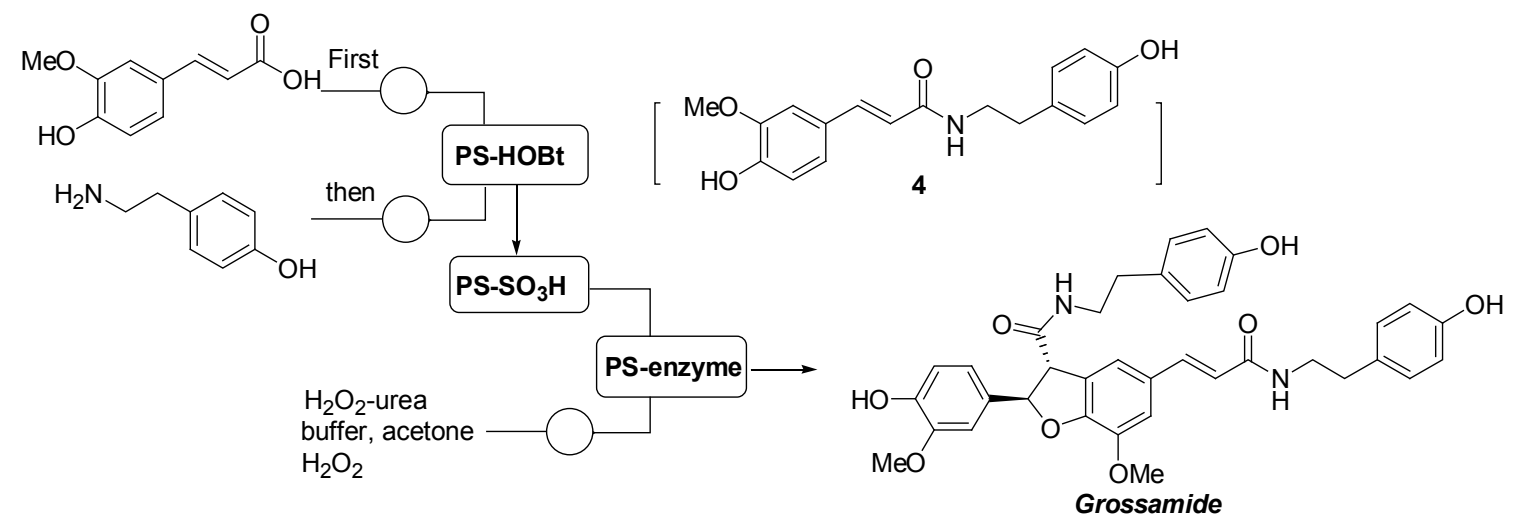

图 2 大海米酰胺的合成

Figure 2 Synthesis of grossamide 
的反应(如有机锂试剂和格式反应等), 由于反应非常剧 烈往往要求在零下数十度甚至更低温度进行. 这一类反 应的共有特征是对温度要求比较严格, 温度高出设定值 会导致副产品生成, 收率和选择性大幅下降. 因此常规 间歇反应一般都采用低温甚至极低温并且使用慢滴加 的方式完成这一过程, 而微反应技术可以很方便地解决 这一难题. 相对于间歇反应, 微反应工艺一般具有以下 几个优势: (1)首先避免了中间副产品的富集进而极大的 提升了反应的收率和安全性; (2) 由于微反应器可以保证 非常短的停留时间(毫秒级), 有效避免了可能的其它副 反应, 因此该微反应工艺可以尝试在较高的温度区间进 行操作而不影响反应的选择性; (3)微反应技术由于在线 的量少可以作为一个工艺快速篮选的平台, 同时在此基 础上也可以实现快速放大, 工艺稳定性良好.

\subsection{1 曲马多的合成}

位于美国 Raritan 的强生药物研发公司的化学发展 和药物评估分部从格氏试剂 3-甲氧基苯基溴化镁和酮 反应制得了产品曲马多(Tramadol), 这是利用该类反应 进行药物中间体连续合成比较早期的例子. 曲马多是一 种鸦片类药物, 作为目前唯一一种中枢性镇痛药, 可缓 解普通到严重的疼痛. 该药是人工合成的, 作用于 $\mu$-阿 片类受体以及去甲肾上腺素和血清张力素系统.

如 Figure 3 所示, 反应生成顺反异构体的比例 (cis/trans)约为 $4: 1^{[22]}$. 过程中生成的有机金属化合物 是不稳定的中间体并且对水汽敏感. 如果使用芳基锂代 替格氏试剂的话, 生成顺式产品的比例更高. 在测定反 应热量的研究中, 3-甲氧基苯基锂的生成和将其加入酮 的这两步工艺都会剧烈放热; 最差情况下的温度上升可 分别达到 62 和 $133{ }^{\circ} \mathrm{C}$. 因此必须将锂中间体保持在很低 的温度下以防止其分解. 在批次反应工艺中, 将 3-甲氧 基苯基锂和环己酮分别在 -10 和 $-65{ }^{\circ} \mathrm{C}$ 下进行操作, 得到相应目标叔醇的产率分别为 $32 \%$ 和 $80 \%$. 考虑连续 反应可以提高反应产率并且降低连续放热造成的安全 隐患, 作者们尝试使用微反应器来替代传统批次反应, 并在微反应器的应用中也充分考虑了温度效应. 由于冷 却器的限制无法尝试更低温度条件而且只有一个微反 应器可用, 因此作者们采用了连续流-批次工艺的组合. 具体工艺为: 金属-卤交换反应在 $-14{ }^{\circ} \mathrm{C}$ 进行(停留时间 为 $17 \mathrm{~s}$ ); 所得锂中间体之后和环已酮于 $-40{ }^{\circ} \mathrm{C}$ 进行批 次反应. 利用这种方法, 在 $87 \%$ 的产率时可以实现 54 $\mathrm{g} / \mathrm{h}$ 的产量.

此外, 来自日本京都大学的 Yoshida 小组 ${ }^{[23 a]}$ 利用微 反应技术在该领域也进行了非常深入的研究, 其中发表 在 Nature 子刊上的工作涉及 “无保护基的有机锂化学” 以及在此基础上发展的天然产物二氢苑酮 Pauciflorol $\mathrm{F}$
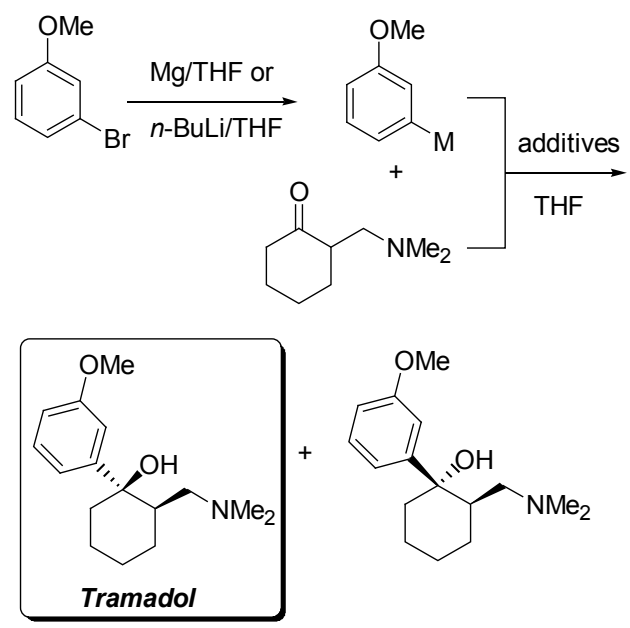

图 3 曲马多的合成

Figure 3 Synthesis of Tramadol

的连续中继合成(formal synthesis)方法学. 尽管有机金 属试剂在药物合成中的应用非常广 (另参见第 2.7.3 节), 由于篇幅所限下文仅再介绍最近发表的抑制剂 AZD6906 的合成工作.

\subsection{2 抑制剂 AZD6906 的合成}

抑制剂 AZD6906 是一个高度官能团化的小分子(其 中包括氨基酮和两性离子的次膦酸), 研究发现有杂质 时存在稳定性问题. 来自瑞典 AstraZeneca 公司研发中 心的研究人员最近将该药物的间歇合成工艺中的前面 两步反应成功地转换为连续流微反应工艺 ${ }^{[23 b]}$, 从而可 以降低成本、提升产品质量(稳定性)以及方便进行工艺 放大(Figure 4).

除了上文提到的稳定性问题，作者们也指出之前间 歇工艺中存在的诸多挑战: (1)反应高度放热, 而且第二 步反应要求严格低温控制以及缓慢滴加操作; 否则产品 会分解或产生副反应; (2)因为商品化来源不足, 反应试 剂二氯甲基膦非常昂贵; (3)在批次工艺中, 由于需要将 烯醇式中间体进行即时质子化以获得产品，因此反应往 往需要过量的碱或其它试剂. 如 Figure 4 所示, 利用连 续微反应技术的优势明显, 可以方便应对并解决绝大多 数的问题: (1)窄通道导致稳定、可控的反应条件, 因此 可以在更高的反应温度下实现对于剧毒试剂的安全操 作，同时方便对该工艺进行优化; (2)利用微型流动反应 器容许使用尽可能少的昂贵试剂以足够的物质的量比 例进行工艺优化, 而且优化后的最佳工艺条件可以通过 简单延长工艺时间、提高原的流速或反应器尺寸进行工 艺放大; (3)由于可以进行连续淬灭, 因此连续反应中使 用的试剂物质的量比例更佳; 而且这样获得的产品纯度 非常高, 解决了之前的稳定性问题并省去后续的柱层析 纯化步骤. 最后的脱保护步骤仍沿用该公司以前开发的 


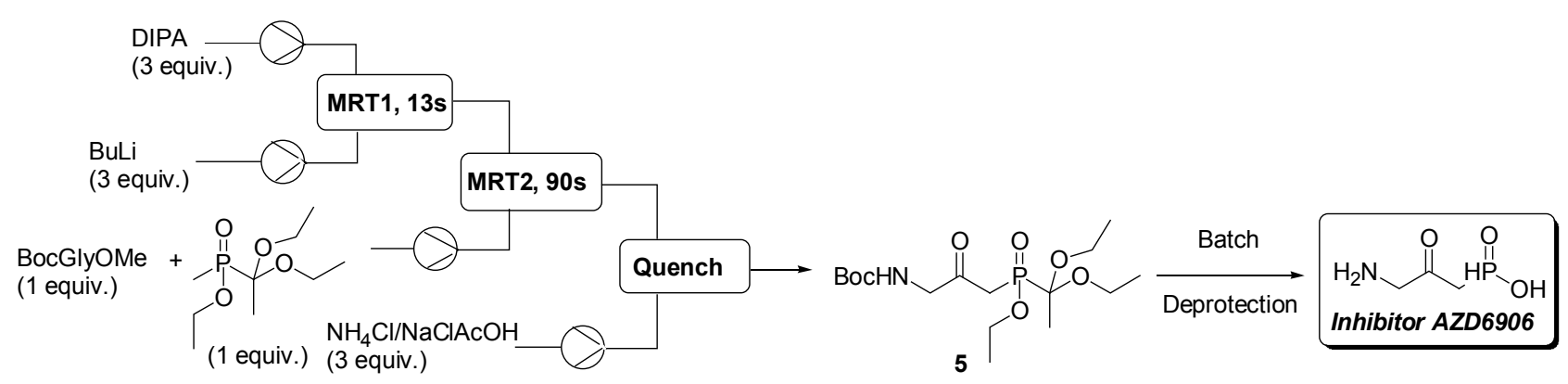

图 4 抑制剂 AZD6906 的合成

Figure 4 Synthesis of inhibitor AZD6906

批次工艺，这样就完成了抑制剂 AZD6906 的高效合成.

\section{3 气一液两相反应}

微反应技术采用由微工程结构组成的不同类型反 应器进行连续处理的新概念 ${ }^{[10,24 a]}$, 因此拓展了传统反 应器的性能, 尤其增强了传质和换热效率. 例如微反应 器可用于快速放热反应, 即使在极端处理条件下和涉及 有害试剂时也可进行安全操作. 气一液微反应器的流体 力学经常被描述为诸如 Taylor 流的均匀流动模式. 一些 气一液微反应器其实就是对应大型反应器的缩小版, 例 如降膜微反应器(详见第 2.3.1 节); 而其它微反应器则利 用了全新的多相接触概念, 例如 Taylor 流或网格微反应 器.

\subsection{1 维生素 D 前体}

拜耳医疗保健(拜耳先灵医药)及其合作者曾经获得 德国公共资助项目 ZOHIR 的资助, 通过微工艺工程联 合开发医药中间体的制备工艺 ${ }^{[24 b]}$. 这些中间体是用于 治疗皮肤疾病和开发基因替代疗法药物所必需的, 一般 为 1 10 t/a 的生产规模. 这项工作的重点是开发和组建 包含连接组件的成套设备, 实现有机化学中的典型反 应, 如臭氧分解、氟化工艺以及澳化/溴氢化工艺等的安 全连续运行. 其中有些反应具有剧烈放热性, 因此需要 通过工艺强化方式来克服其中热转移的局限性. 如果这 些热量突然释放可能会引起爆炸, 因此摚拌批次反应器 中进行的大规模合成只能在低于理想的合成效率条件 下进行. 这样做的结果是开支巨大，甚至一些合成过程 完全不能扩大规模. 而且其中的反应物和产品也都具有
安全危害, 或者具有高活性和热不稳定性. 为了满足臭 氧和卤化工艺操作的安全性要求, 研究人员开发并安装 了小型近红外传感器来进行在线分析和自动化过程控 制 ${ }^{[24 b]}$. 同时为了减少从工艺方案到生产的研发周期(市 场化的时间)，以及降低开发成本和生产设备的安装成 本, 最终开发的微反应器装置采用模块化设计并能够满 足从几千克/年到几吨/年产量范围的不同中间体的生 产.

如 Figure 5 所示, 目标产品维生素 D 前体可以通过 类固醇底物 6 的臭氧分解这类多步气一液反应工艺来制 备, 由于该反应为快速和剧烈放热反应因此使用到降膜 微反应器. 底物 6 中的双键经臭氧化裂解可以形成过氧 化物 7 和醛类中间体 $\mathbf{8}$, 它们随后又被硼氢化钠还原为 目标产品醇 ${ }^{[24 b]}$. 半批次的反应条件是在 $-60{ }^{\circ} \mathrm{C}$ 下通臭 氧 $7 \mathrm{~h}$, 然后淬灭 $1 \mathrm{~h}$; 由于过氧化物积聚的危险性，该 工艺的体积上限为 $10 \mathrm{~L}$. 而使用微反应技术进行该工艺 时，反应可提升至 $-20{ }^{\circ} \mathrm{C}$ 操作，其停留时间仅为 $15 \mathrm{~s}$. (第二步)还原工艺在 $10 \sim 25{ }^{\circ} \mathrm{C}$ 的温度范围内仅需几分 钟即可完成. 所得产品的质量和产量可与小型批次处理 工艺相媲美，产量可达 100 200 g/d.

\subsubsection{2-环戊烯-1,4-二醇的合成}

光化学生成的单线态氧在有机合成中的应用很广 (另见第 2.4 节), 但是富氧有机溶剂及其产品(例如内过 氧化物)的易懪性可能会引起严重危害. 由于微流系统 仅要求很小体积的氧化有机溶剂可以大大降低潜在的 危险, 因此有许多研究小组开始使用微反应器来研究单 线态氧参与的化学工艺过程.
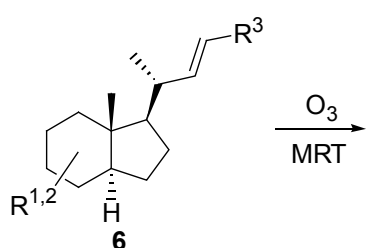

$$
\text { a }
$$

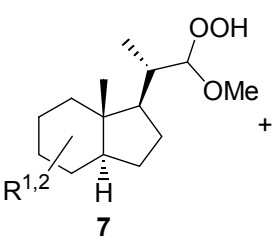

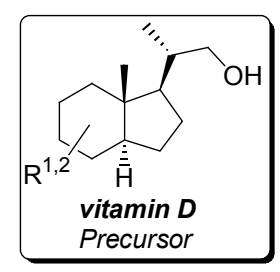

图 5 维生素 $\mathrm{D}$ 前体的合成

Figure 5 Synthesis of vitamin D precursor 
Jähnisch 和 Dingerdissen ${ }^{[25 a]}$ 曾报道利用降膜微反应 器(IMM 制造; 反应板装有 32 个平行微通道, 长 $66 \mathrm{~mm}$ 、 宽 $600 \mu \mathrm{m}$ 、深 $300 \mu \mathrm{m}$ )通过玫瑰红(Rose Bengal)可以生 成单线态氧并和环戊二烯底物 9 发生 [4+2]环加成反应; 其中易爆的内过氧化物中间体被硫䐖很快还原到一种 有重要药学意义的化合物 2-环戊烯-1.4-二醇(10). 而 Seeberger 小组 ${ }^{[25 b]}$ 最近则通过使用四苯基卟啉(TPP)作 为光敏剂实现了同样的高效气一液氧化过程, 以大于 95\%的转化率和 $85 \%$ 的收率获得标题产品. 如 Figure 6 所示, 该工作通过简单、便宜的连续光反应器(构造和降 膜微反应器类似)来现场产生单线态氧, 并适用于各种 烯烃(包括香茅醇，一种香料前体)、1,3-二烯以及硫醚等 的快速氧化. 由于两相混合物在光反应器的窄通道中进 行高速混合可以产生非常大的比表面积, 因此可以实现 高通量而满足制备级的需求, 如该工艺目前的产量可以 达到 $2.5 \mathrm{mmol} / \mathrm{min}$.

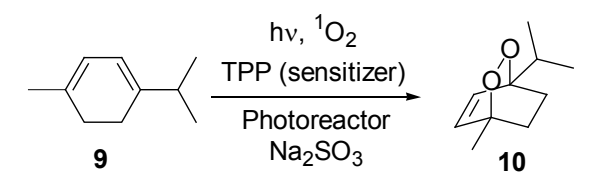

$\left(0.5 \mathrm{~mol} \cdot \mathrm{L}^{-1}\right.$ in $\left.\mathrm{CHCl}_{3}\right) \quad$ (>95\% conv., $85 \%$ yield $)$

图 62 -环戊烯-1,4-二醇的合成

Figure 6 Synthesis of 2-cylcopentene-1,4-diol

\section{4 光反应连续合成}

如前所述, 光化学有机转化对人们来说是一种很有 吸引力的合成方法. 光化学反应由于在反应过程中有非 常活泼的自由基中间体存在，属于强放热或有潜在爆炸 风险的反应类型. 按比例放大光化学反应时就需要考虑
很多因素，例如光源、热和质量传递的测量，以及安全 问题等. 光微反应器有可能克服传统光化学反应器的缺 点, 它最令人感兴趣的几个特点如下: (1)光程极短, 从 而可以广泛而均匀地照射反应混合物; (2)通过调节停留 时间可以避免/最小化不必要的副反应和后续反应; (3) 有效使用光能，可以节能. 已经有不少文献报道利用微 反应技术进行光化学反应以及在这些工艺中相对于常 规反应器的优势 ${ }^{[26 a]}$ ，下文介绍几个在药物合成方面的 例子.

\subsection{1 维生素 D3 的合成}

Takahashi 小组 ${ }^{[26 b]}$ 在 2010 年报道了光微反应器在 药物合成中高效应用的例子，即维生素 $\mathrm{D}_{3}$ 的合成. 如 Figure 7 所示的合成路线也是目前合成维生素 $\mathrm{D}_{3}(\mathrm{Vi}-$ $\operatorname{tamin} \mathrm{D}_{3}$ ) 的工业路线, 从原料维他命原 $\mathrm{D}_{3}$ (provitamin $\mathrm{D}_{3}$ ) 开始经前维生素 $\mathrm{D}_{3}$ (previtamin $\mathrm{D}_{3}$ )中间体的两步光 化学反应. 其中第一步光致开环反应除了生成前维生素 $\mathrm{D}_{3}$ 外, 还同时生成了该中间体的光化学平衡物速甾醇 11 (tachysterol). 虽然第二步反应从前维生素 $\mathrm{D}_{3}$ 到维生 素 D3 是热致 1,7-氢迁移反应，但该小组在加热的油浴 中再增加一个光微反应器; 这样可以促进速甾醇 11 (tachysterol)到前维生素 $\mathrm{D}_{3}$ 的光致平衡移动, 从而再次 实现到维生素 $\mathrm{D}_{3}$ 的热转化, 而最终目的是为了取得更 高的转化率. 因此作者们在优化的流动条件下可以 $32 \%$ 的分离收率(60\%初收率)合成目标产品, 这比目前工业 工艺中一般低于 $20 \%$ 的收率要好不少.

\section{4 .2 青蒿素的合成}

2011 年 9 月 23 日, 有诺贝尔奖 “风向标”之称的 国际医学大奖美国拉斯克奖将其中的年度临床研究奖

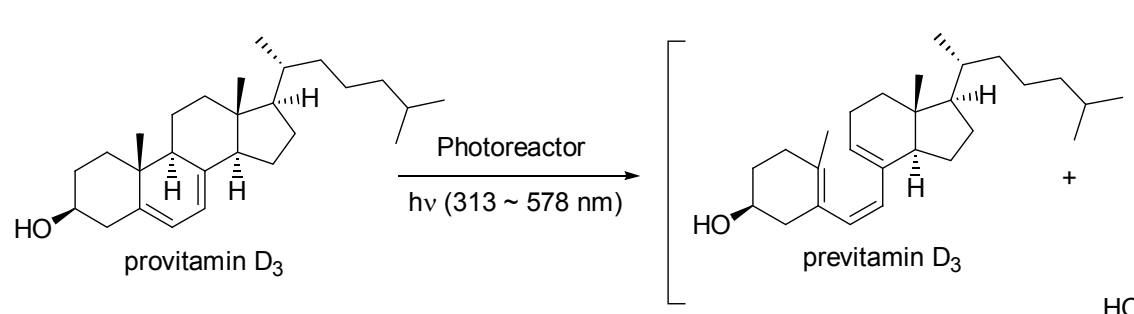

$\frac{\text { Photoreactor, } 100^{\circ} \mathrm{C}}{\mathrm{h} v(360 \mathrm{~nm}), 0.86 \mathrm{~min}}$

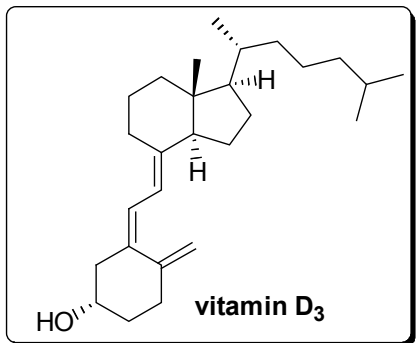

图 7 维生素 $\mathrm{D}_{3}$ 的合成

Figure 7 Synthesis of Vitamin $\mathrm{D}_{3}$ 
授予 81 岁的中国科学家屠呦呦, 以表彰她“发现了青高 素(Artemisinin 或 Qinghaosu)一种治疗㾏疾的药物, 在全 球挽救了数百万人的生命” [27a]. 这是中国科学家首次获 得拉斯克奖, 也是迄今为止中国生物医学界获得的世界 级最高大奖. 青蒿素对鼠症原虫红内期超微结构的影 响, 主要是症原虫膜系结构的改变, 该药首先作用于食 物泡膜、表膜、线粒体, 内质网, 此外对核内染色质也 有一定的影响.

青筒素是从中药青简中提取的有过氧基团的稳定 倍半萜内酯药物, 因此光反应途径是合成该目标分子的 有效方法, 但之前的研究大多采用间歇批次操作工 艺 ${ }^{[27 b]}$. 最近 Seeberger 小组 ${ }^{[27 c]}$ 刚刚报道了从青蒿酸 (Artemisinic Acid)合成青蒿素的高收率、低成本的可放 大连续流工艺. 如 Figure 8 所示, 研究人员从青蒿酸的 氢化反应或以混合酵母的发酵反应中可以方便获得该 合成的关键中间体二氢青蒿酸 (Dihydroartemisinic Acid). 但作者们同时也指出, 后续的三步反应才是该项 工作的难点和挑战之处, 其中包括光化学诱导的 ene 反 应生成中间体 12 (单线态氧)、酸介导的 $\mathrm{O}-\mathrm{O}$ 键的断裂 反应得到中间体 14 (Hock cleavage) 和三线态氧参与的 氧化反应生成中间体 16 ; 而连续流工艺可以提升该工 艺的选择性, 如避免或者最大程度地减少内酯副产品 13 或 15 的生成. 由于该连续流合成工艺无需分离或纯化 其中的中间体而且非常容易进行放大, 因此该工艺的成 功开发实现了技术简便和高效生产。和目前工业上大多 采用从植物中提取分离的方法相比, 从混合酵母中生产 青蒿酸后再通过该工艺获得青蒿素的半合成方法具有 明显优势; 尤其使得该急需的㾏疾药物的生产成本大幅 下降成为可能，而且可以其确保稳定的供应. 基于该小 组已经实现的 $200 \mathrm{~g} / \mathrm{d}$ 的产量, 作者们也推算出仅需要 大约 1500 套类似的简单光微反应器装置就可以满足当
前进行 ACT 治疗用的青蒿素药物.

\section{5 固载催化剂(柱)和固载试剂(柱)参与的连续合成}

由于传统间歇操作中相间接触面积小、传质差等原 因，固载催化剂参与的两相或气一液-固三相反应一般都 受到传质限制，尤其三相反应的反应速率都比较低. 而 流动化学技术由于传质、换热方面优异的过程强化性能 可以有效应对这一挑战，如早期的一个例子是日本东京 大学 Kobayashi 小组 ${ }^{[28 a]}$ 在 2004 年发表在 Science 的工作. 作者们将钯催化剂通过链接基固定在一玻璃毛细管的 内壁从而形成了一固载有钯催化剂的微反应器, 然后对 一些列烯烃、炔烃的还原反应进行了研究. 在优化气相 和液相的流速后，形成了理想的气液环状流现象，三相 的接触面积明显增大. 大多数底物在 $2 \mathrm{~min}$ 即可定量地 转化, 相比常规反应这极大地提升了反应速度. 该微反 应器可以连续使用而不会发生钯催化剂脱落现象，同时 提高了产品的纯度. 在该领域的最新进展之一是由荷兰 Thales Nanotechnology 公司开发的 H-Cube 和 X-Cube 商 品化系统 ${ }^{[28 b, 28 c]}$ 用于非均相氢化和其它转化反应. 由于 已经有许多资料或文献介绍过该技术，本文将不再详细 讨论.

近来在连续多步合成中广泛使用的固载试剂(柱)是 这一领域的另一最新进展. 由于该类固载试剂(柱)可以 在目标工艺结束后通过特定试剂冲洗而实现再生，因 此，该试剂柱与固载催化剂(柱)类似，可以重复多次利 用从而极大地提升了反应效率并降低了工艺成本.

\subsection{1 天然产物 Oxomaritidine 的多步合成}

迄今为止最重要的流动多步全合成之一是来自英 国剑桥大学 Ley 小组 ${ }^{[29 b]}$ 合成生物碱天然产物 $( \pm)$-oxomaritidine 的工作. 之前该小组已经通过间歇工艺以 5 步 反应、 $62 \%$ 的总产率合成了该目标分子，而且其中只

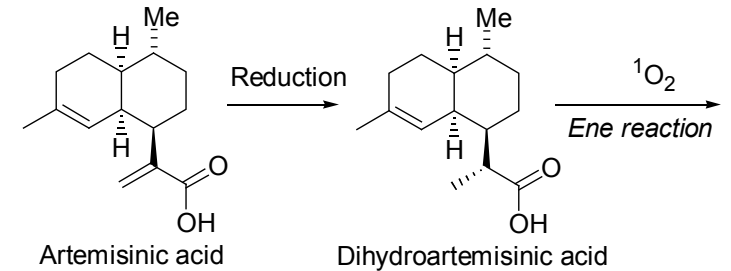

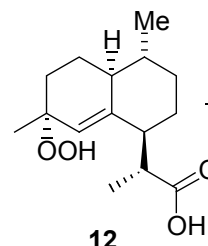
Hock cleavage

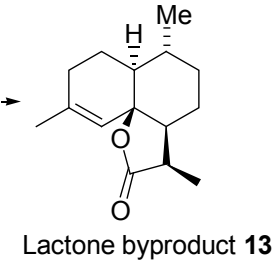

Lactone byproduct 13

$\checkmark$
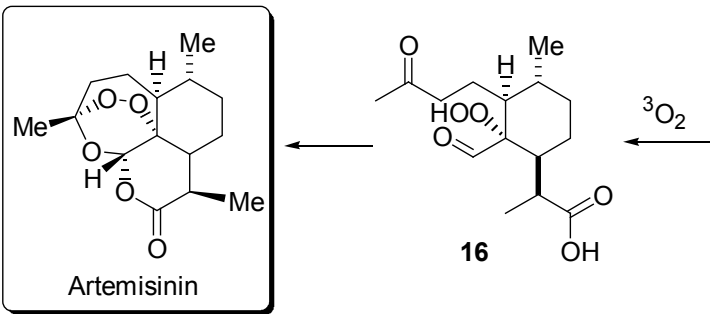<smiles>CC(=O)CC[C@H]1/C(=C\O)[C@@H](C)CC[C@H]1C(C)C(=O)O</smiles>

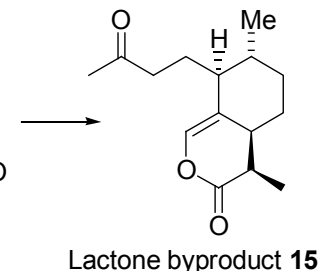

图 8 青蒿素的合成

Figure 8 Synthesis of artemisinin 
使用了固载试剂 ${ }^{[29 a]}$, 因此这被认为是进行流动合成尝 试的有趣对照. 如 Figure 9 所示, 该合成体现了流动化 学的许多优点. 首先, 具有潜在毒性和爆炸性的叠氮生 成后立即与 $\mathrm{PS}-\mathrm{PBu}_{2}$ 柱反应形成相应的聚合物负载的氮 杂 Wittig 反应的中间体; 这样可以防止人接触到叠氮, 并可以减缓膦亚胺的形成而控制氮气的释放. 然后通过 在线切换开关在该 $\mathrm{PS}-\mathrm{PBu}_{2}$ 柱中引入另一股含有(流动) 氧化产物的液流, 反应后得到所需要的亚胺. 该亚胺中 间体的液流经 H-Cube (另见第 2.5.2 节) 而发生连续流动 氢化反应生成二级胺中间体 17. 之后利用 V-10 溶剂蒸 发器去除四氢呋喃并将其溶解在二氯甲烷中, 这一操作 在不到 $10 \mathrm{~min}$ 内可以完成. 最后序列中的反应包括: 将 玻璃微流芯片加热至 $80{ }^{\circ} \mathrm{C}$ 后从三氟乙酸䣶(TFAA)得到 三氟乙酰基保护的中间体 18 (过量的三氟乙酸䣶可以使 用 QP-BZA 柱来去除); 该反应流导入 PS-PIFA 柱中发 生酚的氧化偶联得到含七元环的三环中间体; 然后该溶 液通过氢氧离子交换树脂 $\mathrm{PS}-\mathrm{NMe}_{3} \mathrm{OH}$ 柱进行二级胺的 脱保护后随即自发成环. 最终以 $90 \%$ 的纯度合成目标化 合物 $( \pm)$-oxomaritidine, 整个步骤需要约 $6 \mathrm{~h}$ 完成; 这与 常规批次反应条件下估计需要 $4 \mathrm{~d}$ 的批次反应时间形成 了鲜明对比. 作者们发现合成总产率为 $40 \%$ (可重复); 而其中酚氧化步骤是产率限制步骤(仅获得 $50 \%$ 的目标 产品), 其它步骤的产率几乎定量.

通过以上例子我们可以注意到固载试剂参与的合 成和流动合成方法有许多不同点. 已经报道的合成不能 自动转化成流动合成, 这说明溶液相、固载试剂和流动
化学均有各自的复杂性. 这将需要研究人员花费时间来 优化和有效地利用这些不同的技术. 下面的例子是最近 发表在 Chem. Commun. 上固载试剂(柱)参与的伊马替尼 合成工作.

\subsection{2 伊马替尼的合成}

由瑞士诺华公司推出的药品 “甲磺酸伊马替尼 (Imatinib Mesylate)”, 商品名为 “格列卫(Gleevec)”[30a,b]. 该药用于治疗慢性骨䯣性白血病和胃肠基质肿瘤，治疗 效果得到了广泛认可. 甲磺酸伊马替尼在体内外均可在 细胞水平上抑制 Bcr-Abl 酪氨酸激酶，能选择性抑制 Bcr-Abl 阳性细胞系细胞、 $\mathrm{Ph}$ 染色体阳性的慢性粒细胞 白血病和急性淋巴细胞白血病病人的新鲜细胞的增殖 和诱导其调亡. 此外, 甲磺酸伊马替尼还可抑制血小板 衍化生长因子(PDGF)受体、干细胞因子(SCF), c-Kit 受 体的酪氨酸激酶, 从而抑制由 PDGF 和干细胞因子介导 的细胞行为.

该项工作同样来自英国剑桥大学的 Ley 小组 ${ }^{[30 c]}$, 他们通过使用一系列聚合物负载的试剂和催化剂柱, 经 3 步流动合成以 $32 \%$ 的总收率 $(95 \%$ 纯度)获得目标药物 中间体伊马替尼(Imatinib). 如 Figure 10 所示, 上述的不 同反应步骤包括酰胺中间体 19 的合成、亲核取代反应 生成中间体 20 以及钯催化的中间体 20 和 21 的 Buchwald-Hartwig 偶联反应.

毫无疑问，固载物种的引入大大拓展了流动化学在 复杂药物中间体或高纯度产品生产中的应用. 然而该领

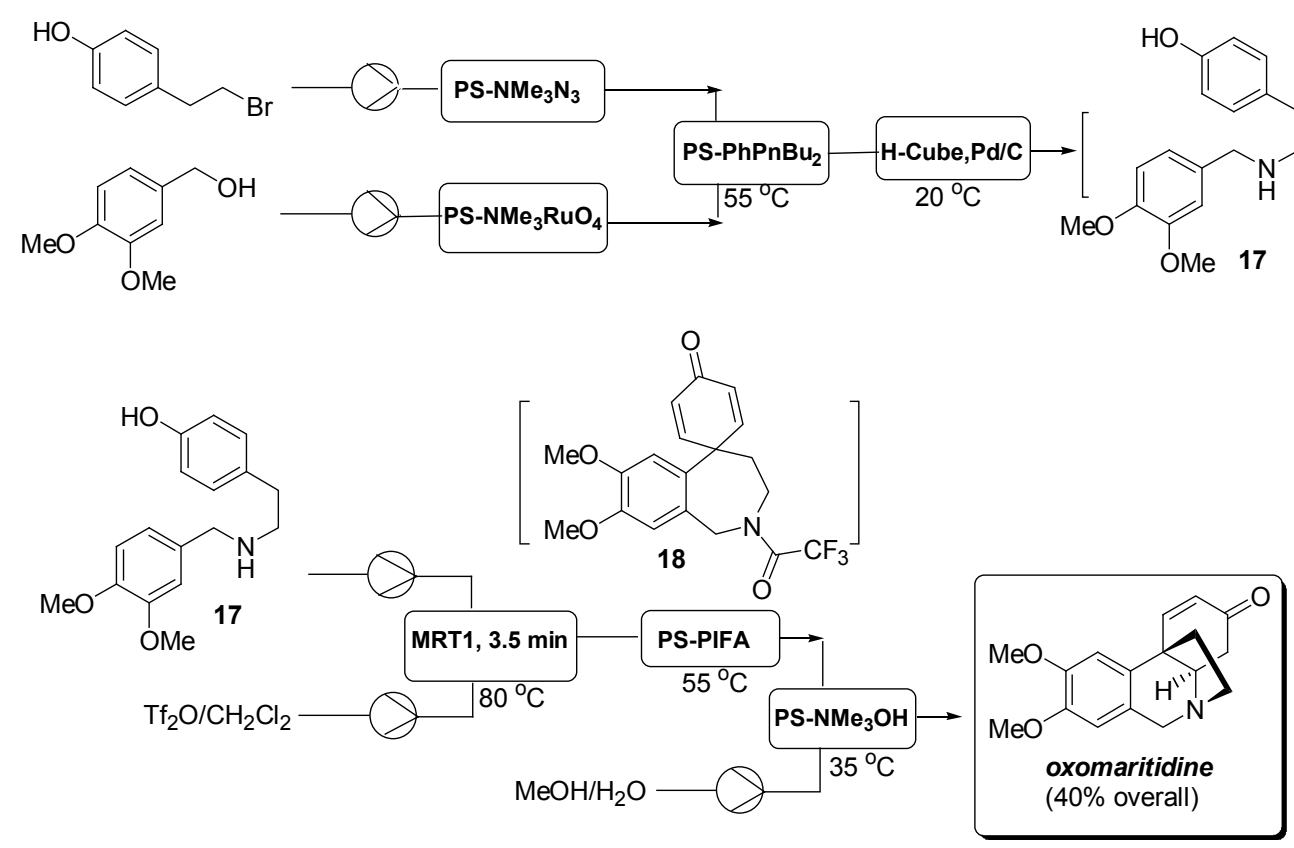

图 9 天然产物 Oxomaritidine 的多步合成

Figure 9 Multistep synthesis of natural product oxomaritidine 


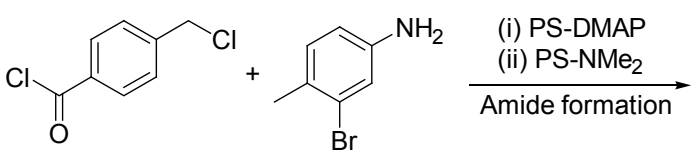<smiles>Cc1ccc(NC(=O)c2ccc(CCl)cc2)cc1Br</smiles>

$\mathrm{CaCO}_{3}$

1-methylpiperazine

PS-NCO

Nucleophilic substitution<smiles>Cc1ccc(NC(=O)c2ccc(CN3CCN(C)CC3)cc2)cc1Br</smiles>
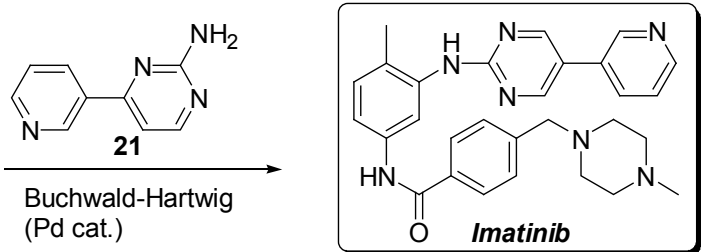

图 10 伊马替尼的合成

Figure 10 Synthesis of Imatinib

域的继续发展还面临着许多挑战, 特别是需要考虑不同 反应间的兼容性或者需要中间体分离纯化步骤的连续 多步复杂流动合成(参见第 2.7 节).

\section{6 微波技术与连续流合成}

微波作为一种传输介质和加热能源已经被广泛应 用于有机合成、无机合成、分析化学、非均相催化、采 油、炼油、治金、环境污染治理等众多化学研究领域 ${ }^{[31 a]}$. 与常规的加热方法不同, 微波辐射是表面和内部同时进 行的一种体系加热. 由于无需热传导或对流因而不产生 温度梯度, 因此可以将体系迅速升温但受热均匀. 微波 促进的有机反应其优势体现为可缩短反应时间、提高反 应的选择性和收率、减少溶剂用量甚至可以进行无溶剂 反应，还可以简化后处理过程而减少三废，因而也被列 入 “绿色化学” 的一种 ${ }^{[31 b]}$. 前面提到, 连续流合成在其 发展过程中也在不断与其它相关辅助技术进行联合或 集成, 微波技术就是其中一个很好的例子. 由于同样属 于过程强化技术，在连续流工艺开发前期通常可以首先 利用微波促进的反应来进行工艺条件的初步测试和篎
选，即“微波到流动合成” 优化模式 ${ }^{[32 \mathrm{c}-\mathrm{f}]}$.

\subsection{1 杀真菌剂啶酰菌胺的合成}

巴斯夫公司研发的杀真菌剂啶酰菌胺(Boscalid $\left.{ }^{\circledR}\right)$ 属 于食品内/表的杀菌剂 ${ }^{[32 a]}$. 奥地利的 Kappe 小组 ${ }^{[32 b]}$ 于 2010 年发表了啶酰菌胺的流动合成工作，其中将微波反 应技术与流动化学有机地结合在一起. 依照该小组之前 的 “微波到流动合成” 的优化模式 ${ }^{[32 e, 32 \mathrm{f}]}$, 作者们首先使 用间歇微波加热技术(封管)对于如 Figure 11 所示的所有 3 步反应进行了小规模试验，其最终目标是通过过程强 化尝试在尽可能短的时间内来实现高通量连续流动合 成. 基于微波技术取得的初步结果，研究人员很快成功 开发了前两步的连续流工艺, 并以高选择性和良好收率 获得合成啶酰菌胺的重要中间体胺 23.

如 Figure 11 所示, 该两步连续工艺包括钯催化的 Suzuki-Miyaura 偶联反应(微管流反应器)和铂/碳催化的 高选择性非均相氢化反应，之后进行酰胺化就可以得到 目标产品啶酰菌胺. 其中在第二步还原硝基的氢化反应 工艺中，作者们利用荷兰 Thales Nanotechnology 公司开

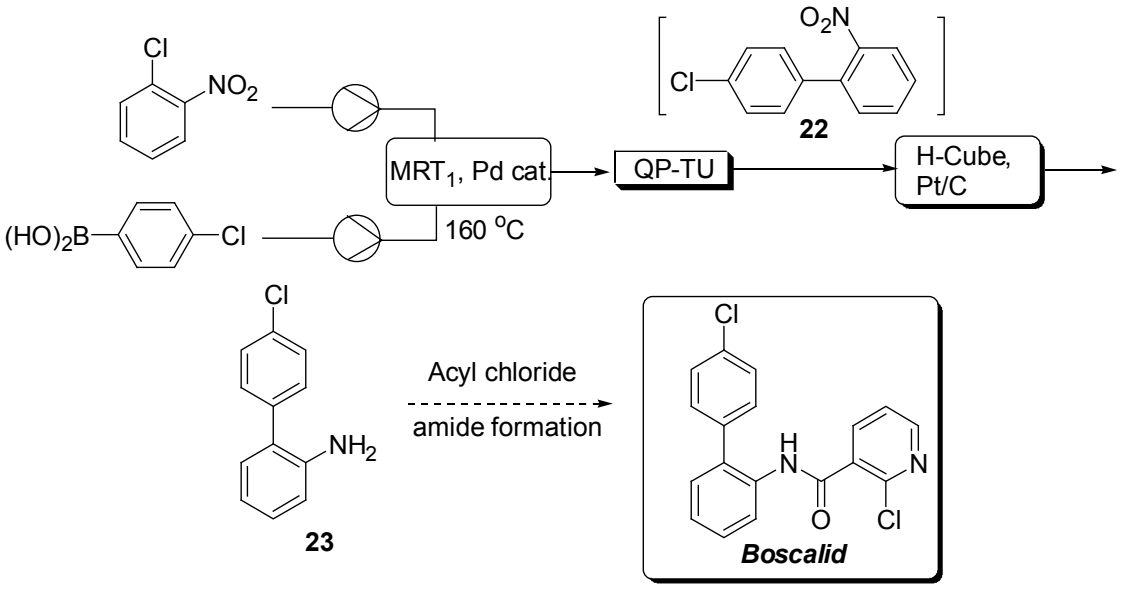

图 11 杀真菌剂啶酰菌胺的合成

Figure 11 Synthesis of fungicide Boscalid ${ }^{\circledR}$ 
发的 H-Cube 技术(另见第 2.5 节)成功笁选出非均相铂催 化剂, 可以实现硝基的高选择性还原而不影响中间体 22 的氯取代基(钯催化剂的化学选择性不理想). 由于这两 步工艺中使用的金属催化剂不同，作者们在这两步反应 工艺之间增加了一硫脲基的树脂柱(QP-TU)在线纯化硝 基联苯中间体 22, 以防第一步工艺中用到的钯金属发生 渗漏而对第二步工艺产生不利影响. 结果可以证明该两 步流动工艺在实验室级别合成的可行性概念, 后续的工 艺放大以及第一步工艺中钯催化剂的回收实验还在进 一步研究中.

\subsection{2 顺铂的合成}

顺铂(Cisplatin, [cis- $\mathrm{PtCl}_{2}\left(\mathrm{NH}_{3}\right)_{2}$ ]) 是一种含铂的抗癌 药物, 即顺式-二氯二氨合铂(II). 顺铂是棕黄色粉末, 属于细胞周期非特异性药物，对肉瘤、恶性上皮肿瘤、 淋巴瘤及生殖细胞肿瘤都有治疗功效. 它是一大类铂类 药物中最早被合成的一个, 结构最简单且作用机理明 确; 它的发现又导致了其他抗癌药物的研制, 包括卡 铂、奥沙利铂、奈达铂及赛特铂等. 早期合成顺铂的反 应路线主要有收率低、耗时长以及工艺的不可靠性等缺 点. 尽管随后的改进路线对于这些问题有所改善, 但仍 然存在反应选择性方面的挑战, 即化学选择性(副产品 Magnus 盐的生成, $\left[\mathrm{Pt}\left(\mathrm{NH}_{3}\right)_{4}\right]\left[\mathrm{PtCl}_{4}\right]$ ) 和顺反选择性(只有 顺式异构体是目标产品 $)^{[33 a-b]}$.

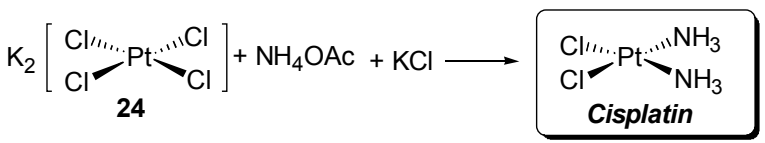

图 12 顺铂的合成

Figure 12 Synthesis of cisplatin

Leadbeater 等 ${ }^{[33 c]}$ 于 2011 年发表了连续流合成顺铂 的工作, 最佳工艺条件下的总收率为 $72 \%$, 而且没有副 产品如 Magnus 盐的生成. 作者们首先使用微波技术进 行小规模的反应条件优化, 可以在快速高效的合成顺铂 并取得非常好的化学选择性(没有发现 Magnus 盐的生 成); 但是当该间歇微波工艺放大到克级水平时，反应的 放大效应就很明显(产生了大量的副产品 Magnus 盐). 如 Figure 12 所示, 作者们随后考虑改用连续流工艺进行 工艺放大，同时针对目标产品溶解性的问题进行了浓度 和其它工艺条件的调整。最优化的结果是以 0.8 $\mathrm{mL} / \mathrm{min}$ 的流速将物料流经连续反应器的加热区域, 收 集到第一批产品( $45 \%$ 收率)后将滤液再通过该连续反应 器又可获得第二批产品(26\%收率); 而且没有任何堵塞 或副产品生成的现象.

\section{7 连续多步流动合成}

虽然连续流工艺的特点非常适合多步反应的自动
化合成, 但由于多步反应体系变得非常复杂因而也更具 有挑战性，如涉及到的试剂众多而且每步反应的条件 (包括溶剂、温度和压力等)可能均有不同变化. 下文将 重点介绍流动化学技术应用于多步复杂合成体系中需 要注意的问题、应对方法以及相关辅助技术的最新进 展 ${ }^{[17 \mathrm{~d}]}$, 其中列举的例子涉及反应体系的兼容性、溶剂更 换、连续工艺和间歇工艺的集成、在线检测技术、在线 纯化技术(固载试剂柱)以及化合物库的合成(组合化学) 等几个方面.

\subsection{1 布洛芬的合成}

布洛芬(Ibuprofen)具有抗炎、镇痛、解热作用，适用 于治疗风湿性关节炎、类风湿性关节炎、骨关节炎、强 直性脊椎炎和神经炎等，但治疗风湿和类风湿关节炎的 疗效稍逊于乙酰水杨酸和保泰松.

2009 年 McQuade 小组 ${ }^{[34]}$ 成功开发了合成非甾类抗 炎药物布洛芬的三步连续微反应工艺, 其中可以省去中 间体的纯化和分离步骤. 如 Figure 13 所示, 第一步工艺 为傅克酰化反应，由纯的异丁苯、丙酸以及三氟乙酸在 $150{ }^{\circ} \mathrm{C}$ 混合 $5 \mathrm{~min}$ 后即可完成; 傅克反应后的中间体 $\mathbf{2 5}$ 随后与第二步工艺物料中原酸三甲酯(TMOF) 和醋酸碘 苯发生 1,2-芳基迁移反应生成目标中间体 26. 最后一步 工艺通过将碱液流引入体系而实现㿝化反应，最终产品 布洛芬可以通过柱层析(70\%收率)或简单重结晶(51\%收 率)进行纯化. 虽然当前可以实现的产量大约为 9 $\mathrm{mg} / \mathrm{min}$ 粗产品, 作者们也提到通过平行增加反应器数 量、增长通道长度以及使用长效洜入系统可以有效地进 行工艺放大实现高通量连续合成. 同时利用微反应技术 进行工艺放大也具有在不同反应步骤(如从 $150{ }^{\circ} \mathrm{C}$ 到 30 ${ }^{\circ} \mathrm{C}$ 的不同反应温度)中仍可以保持精确控温的优势; 而 在大规模的间隙工艺中却难以进行类似的连续操作并 能保证每一步反应温度条件的有效控制.

值得一提的是，本文对于多步连续反应工艺中反应 体系的兼容性也进行了研究. 由于希望该工艺可以省去 中间体纯化的步骤, 要求在前面步骤中产生的副产品和 过量的试剂必须和下游反应是兼容的. 因此，作者们从 反合成分析开始就把该连续合成工艺当作一个整体研 究, 而不是把它们看作相互独立的一系列反应步骤. 体 系兼容性的问题解决之后, 该多步工艺就可以连续进行 而不存在中间环节来打断它. 例如通过对照实验，作者 们发现在第一步傅克酰化反应中过剩的试剂(三氟乙酸 和未反应完全的异丁苯)不影响第二步中的 1,2-芳基迁 移反应, 相反地可以促进该反应而无需再添加其它酸.

\subsection{2 天然产物 Siphonazole 的合成}

来自英国剑桥大学化学系的 Ley 小组 ${ }^{[35]}$ 于 2011 年 发表了含双噁唑结构天然产物 $O$-甲基 Siphonazole 的新 


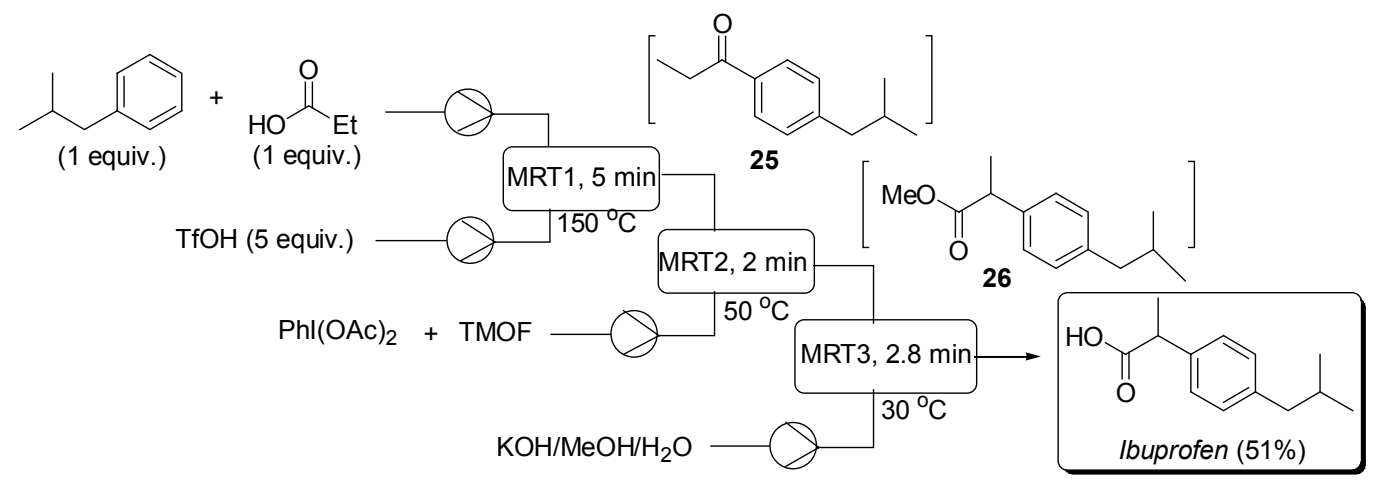

图 13 布洛芬的合成

Figure 13 Synthesis of ibuprofen

型全合成工艺，其中集成了不同阶段的连续微反应工艺 和间歇工艺; 该模块化合成工艺的成功开发也为非天然 的 Siphonazole 类似物的合成提供了方便的路线. 同时 该合成中也成功使用了固载试剂柱的在线纯化技术, 这 样可以避开不必要的传统分离纯化步骤而提升整个合 成的效率. 该合成的反合成分析可以追踪到噁唑单元 29 和 $30 \mathrm{a}$ 以及共轭烯丙胺前体, 其中的关键合成步骤依次 为 Claisen 缩合反应、Krapcho 去羰基化反应和水解、酰 胺化反应; 该工作最终以 9 步的总步数合成了目标天然 产物 $O$-甲基 Siphonazole.

如 Figure 14 所示, 该合成包括两段连续微反应工艺 和两段间歇工艺: (1)从二甲氧基肉桂酸到噁唑啉衍生物 27、再氧化到噁唑着酸衍生物 28 的连续工艺; 在这两步 微反应工艺中均使用非均相固载试剂柱实现了在线纯 化并可提升整个工艺体系的兼容性, 其中包括负载磺酸 柱 QP-SA、负载叔胺柱以及碳酸钲/二氧化硅填料柱; (2) 从噁唑着酸衍生物 28 到啞唑酰氯衍生物 29 的间歇工艺; (3)聚合物负载的碱催化的连续 Claisen 缩合反应工艺, 即从 29 和 $30 \mathrm{a}$ 出发合成烯醇式中间体 $31 \mathrm{a}$, 随后水解为 中间体 32a; (4) Krapcho 去羰基化间歇反应得到中间体 $33 \mathrm{~b}$ 及随后的一锅法水解、酰胺化间歇反应工艺, 其中 去羰基化反应中还使用了微波加热技术. 作者们强调该 合成成功之处在于有机地集成了连续流技术和传统的 间歇工艺，同时在连续反应工艺之间应用固载试剂柱进 行在线纯化也极大地提升了整个工艺的效率.

\section{7 .3 珠氯噻醇中间体的合成}

丹麦 H. Lundbeck A/S 公司开发的药物珠氯噻醇 (zuclopenthixol)可增强酒精的镇静作用、巴比妥类药物 及其它中枢神经系统抑制剂的作用. 最近该公司及其合 作者 ${ }^{[36]}$ 报道了合成珠氯噻醇的乙烯基乙醇关键中间体 的连续流工艺, 其中应用的在线近红外分析技术(NIR) 对于提升该格氏试剂参与的烷基化反应不论在工艺自 动化控制、在线监测以及放大生产等方面都具有潜在优
势. 如 Figure 15 所示，作为珠氯噻醇整个合成工艺的第 一步反应，酮底物 34 和格氏试剂的烷基化反应首先生 成活泼中间体 35 , 然后水解得到乙烯基乙醇关键中间 体. 作者们发现, 为使杂质的产生达到最小化, 要求使 用低于或等于底物酮 34 化学计量的格式试剂; 但另一 方面格氏试剂用量过低也会造成反应收率的下降. 因此 对于格氏试剂和底物酮 34 剂量的精确控制成为该工艺 取得成功的关键. 为实现这一目的并能够完全自动控 制，作者们采用了前馈一反馈(feedforward-feedback )的 控制系统，其中在反馈控制中作者们使用流动池单元对 于整个反应体系进行在线近红外分析. 不同于费时、费 力的离线高压液相分析技术 (多数情况下还需要将样品 进行稀释等过程)，在线近红外技术可以实时监测反应 体系并能够通过有效的数据模型提供更加快速且丰富 的信息量, 这样为工艺的自我调节和实时释放开辟了新 的方向. 在该研究中值得关注的具体环节包括以下几个 方面: (1)首先, 作者们为获取正确的进料量比例创建了 化学计量模型的系统性方法; (2)使用近红外技术可以在 真实的工艺条件下对于高活性的格氏试剂进行在线监 测，而且分析响应的时间足够短，因此可以提供反应体 系的实时动态信息; (3)工艺放大至生产级别时，同样可 以使用该在线监测技术追踪反应物的消耗情况; 同时可 以对于工艺过程中出现的反常情况进行鉴别，例如格氏 试剂过量、试剂的突然堵塞或者是连续流工艺出口处可 能的产品结晶等等.

\subsubsection{1,2,3-三唑的合成}

因为在从细胞生物学到材料科学的许多不同化学 领域都有应用，以铜(I)介导的有机叠氮化合物和末端炔 烃偶联合成三唑(Triazole)的反应目前引起了广泛兴 趣 ${ }^{[37 a]}$. 其批次反应通常涉及从反应溶液中结晶获得产 品, 但这样会经常夹杂大量的铜催化剂, 因此在得到化 合物纯品前需要进一步分离. 2009 年 Ley 小组 ${ }^{[37 b]}$ 发表的 工作充分说明，在连续流反应体系中引入固载试剂的 
(1)

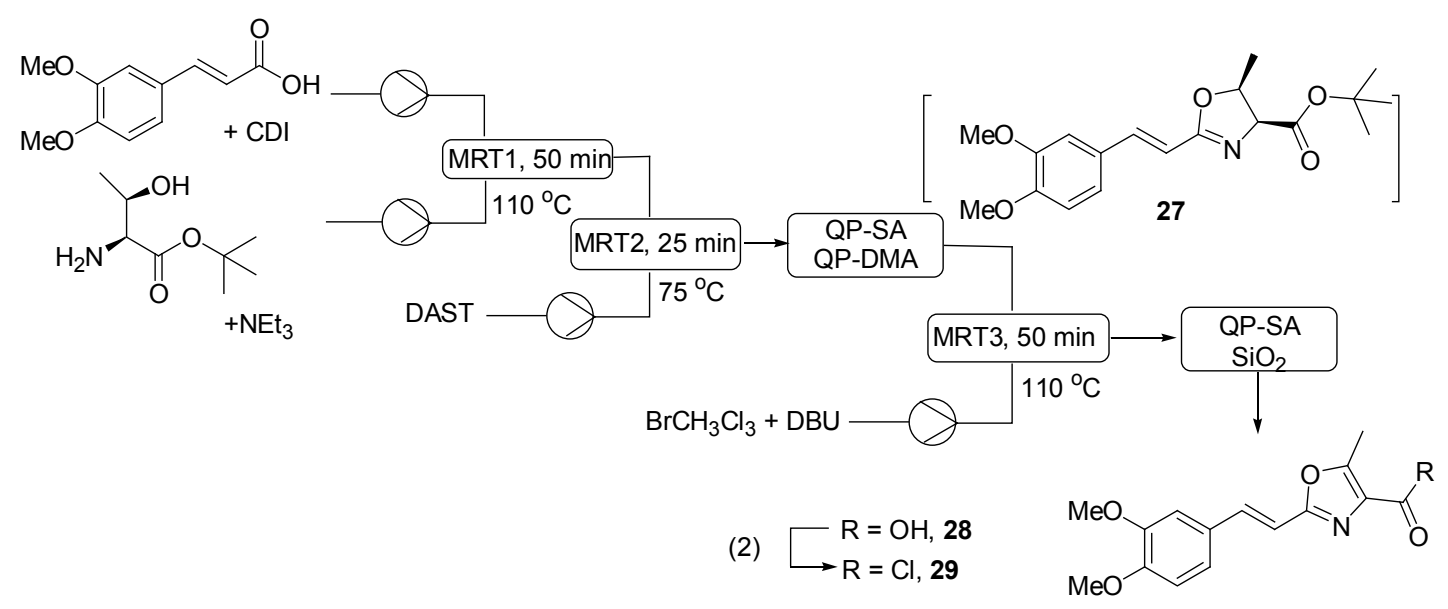

(3)

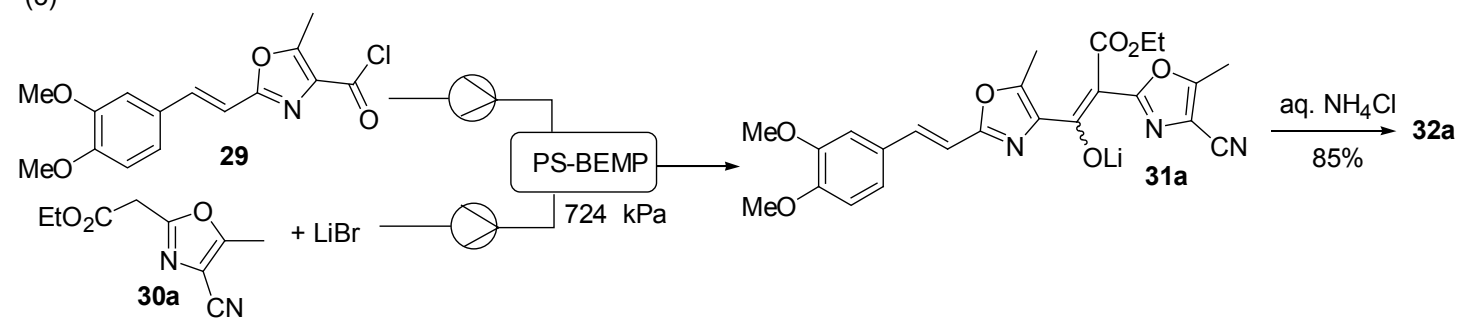

(4)<smiles>[R]C#[R16]c1ccc(OC)c(OC)c1</smiles><smiles></smiles>

图 14 天然产物 Siphonazole 的合成

Figure 14 Synthesis of natural product siphonazole

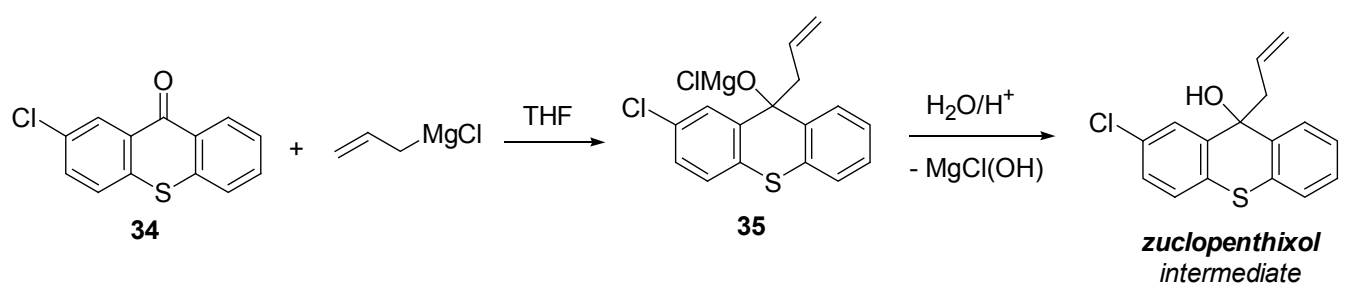

图 15 珠氯噻醇中间体的合成

Figure 15 Synthesis of zuclopenthixol intermediate

在线纯化技术可以高效进行多组分参与的多步偶联反 应获得高纯度的三唑产品, 而无需进行中间体的后处理 过程. 整个工艺包括使用 Bestmann-Ohira 试剂从底物醇
制备末端炔类中间体以及铜介导的该炔烃中间体和叠 氮化合物偶联合成三坐. 如 Figure 16 所示, 将叠氮化合 物 36、底物醇 $\mathbf{3 7}$ 以及 Bestmann-Ohira 试剂 $\mathbf{3 8}$ 的混合物 


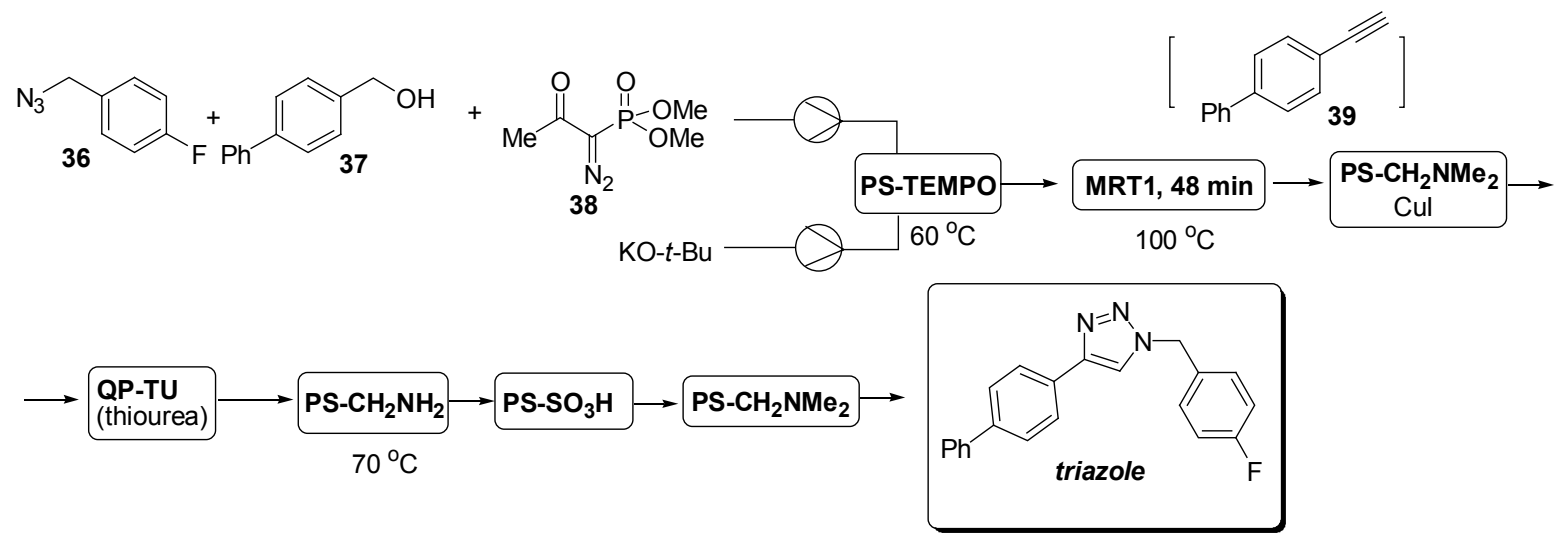

图 16 1,2,3-三唑的合成

Figure 16 Synthesis of 1,2,3-triazoles

一起流经预先活化的 PS-TsOTEMPO 柱, 醇 37 首先发生 原位氧化得到醛中间体而不影响混合流中的其它成分; 随后该醛中间体和叔丁醇钾的溶液混合并进入微反应 器中反应 $48 \mathrm{~min}$ 生成末端炔中间体 39. 偶联环化反应 通过预载碘化铜(I)的树脂柱催化完成, 产生的液流导入 四根串联的试剂柱(次序为 QP-TU 柱、QP-BZA 柱、负 载磺酸基柱和负载二甲氨基柱)进行在线纯化, 最终以 $55 \%$ 的收率、大于 $95 \%$ 的纯度完成了该单程连续多步流 动合成.

\subsubsection{Camalexin 化合物库的合成}

由于吲哚基噻唑类化合物(Tadalafil, Cmalexin 和 BE 10988 等)在治疗领域的广泛应用 ${ }^{[38 a ~ 38 c]}$, 其高通量 的合成方法一直是研究人员的兴趣所在. 美国 SanfordBurnham 药物研究所的 Cosford 小组 ${ }^{[38 d]}$ 最近报道了 2-(1H-吲哚-3-烃基)噻唑衍生物的连续流多步自动合成. 如 Figure 17 所示, Hantzsch 噻唑合成中间体 43、去缩酮 化反应以及 Fischer 吲哚合成多步连续工艺可以快速有 效地提供高度官能团化的 Camalexin 类化合物库. 通过 该工艺可以在 $15 \mathrm{~min}$ 时间内以高收率 $(38 \% \sim 82 \%)$ 获得 这
些类似药物的复杂小分子，而无需中间体的分离步骤. 此外，作者们也开发了一锅法的间歇工艺合成相关的 2氢一吲哚基噻唑类化合物库.

随着易于改装的模块化流动反应器组件的商品化, 连续多步流动合成工艺的发展非常迅速. 一方面, 实验 室设备的变革和灵活组装以及进行积木式的按需合成 正创造出新的工作环境; 另一方面，对于原子经济性反 应的追求和尽可能少使用有机溶剂的要求正改变着人 们的态度并刺激着合成技术的不断创新. 流动化学尘然 会增强药物研发人员的技能和创新能力; 然而这些设备 只有在操作者的熟练掌握下才有效，不应被视为人力的 替代.

\section{8 其它流动合成}

其它流动合成的例子请参见图 18.

\section{3 结语和展望}

综上所述, 流动化学近十年来在药物合成领域已经 取得非常大的进展. 越来越多的知名国际制药企业开始 公开发展该技术，而且文献中也出现了不少连续多步流
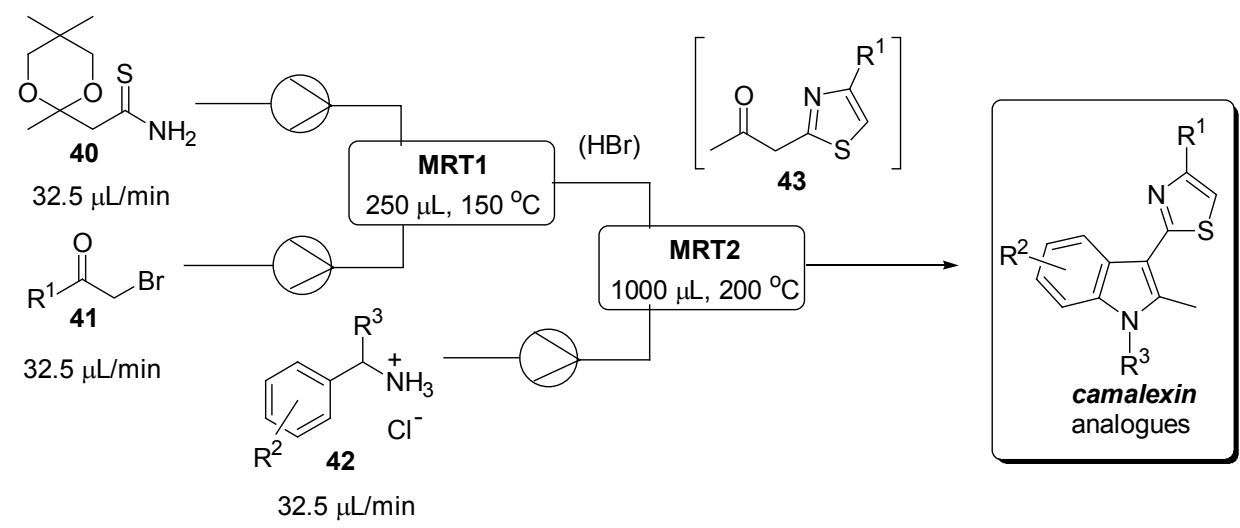

图 17 Camalexin 化合物库的合成

Figure 17 Library synthesis of camalexin analogues 
<smiles>CC(=O)OC/C=C(C)/C=C/C=C(C)/C=C/C1=C(C)CCCC1(C)C</smiles>

Vitamin $\mathbf{A}^{[39]}$

Karge, 2002

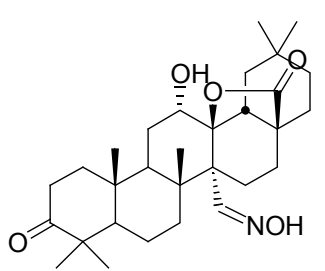

Endothelin receptor antagonist ${ }^{[42]}$ Ryu, 2006<smiles>CCOCCOc1ccc(CC(=O)Nc2cc(C)cc(C)c2)cc1</smiles>
Seeberger, 2008<smiles>CC(O[C@@H]1OCCN(Cc2n[nH]c(=O)[nH]2)[C@H]1c1ccc(F)cc1)c1cc(C(F)(F)F)cc(C(F)(F)F)c1</smiles>

MK869 $^{[47]}$

Eli Lilly, 2009<smiles>CC(C)CCCC(C)CCCC(C)CCCC(C)C</smiles>

Pristane $^{[50]}$

Fukase, 2009<smiles>COc1cc(C2CCCN(C)CC2)c2[nH]c(C(=O)Nc3ccc(N4CCOCC4)cc3)cc(=O)c2c1</smiles>

Quinolone derivative ${ }^{[53]}$ Ley, 2010<smiles>O=C(O)c1cn(C2CC2)c2cc(N3CCNCC3)c(F)cc2c1=O</smiles><smiles>CO/N=C1\CN(c2nc3c(cc2F)c(=O)c(C(=O)O)cn3C2CC2)C[C@H]1CN</smiles>

LG, 2005<smiles>CN(C)CCCC1(c2ccc(F)cc2)OCc2cc(C#N)ccc21</smiles>

Citalopram $^{[43]}$ Pitts, 2006<smiles>Cc1c(C(=O)NN2CCCCC2)nn(-c2ccc(Cl)cc2Cl)c1-c1ccc(Cl)cc1</smiles>

Seeberger, 2008

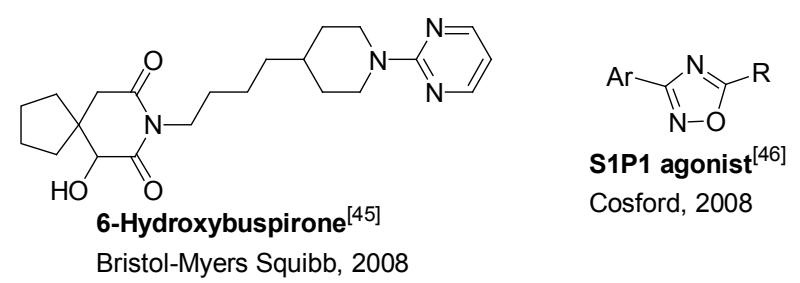

$\mathrm{HO}$,<smiles>C=C[C@](C)(O)CCC=C(C)C</smiles>

Linalool $^{[48]}$

Buddoo, 2009

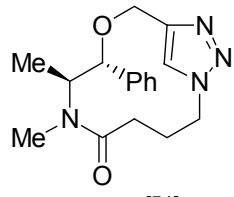

Macrocyle $^{[51]}$

Bogdan, 2010

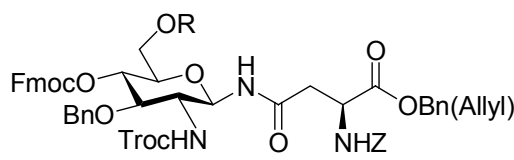

N-glycopeptide ${ }^{[49]}$

Fukase, 2009

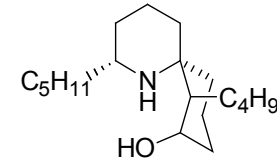

Perhydrohistrionicotoxin ${ }^{[54]}$ Holmes, 2010

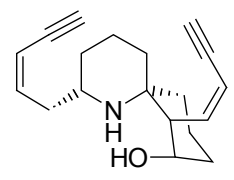

Histrionicotoxin ${ }^{[52]}$ Brasholz, 2010<smiles>CCN(CC)C(=O)c1ccc(C(=C2CCNCC2)c2cccc(F)c2)cc1</smiles>

a-Opioid receptor agonist ${ }^{[55]}$ Ley, 2010

图 18 其它流动合成

Figure 18 Other examples of flow synthesis

动合成药物中间体的成功案例. 可以看出, 实现多步流 动合成的关键在于连续毗邻反应间的工艺衔接技术或 者反应中间体的在线分离/纯化技术, 已经报道的包括 固载试剂柱在线纯化、连续萃取或膜分离技术 ${ }^{[56]}$ 和连续 色谱技术等. 同时以流动化学的概念对于多步合成工艺
过程进行整体反合成分析或重新设计也是目前的选项 之一.

虽然流动技术并不能完全替代间歇工艺，而且在成 为药物研发和合成的通用方法之前流动化学仍需要应 对不少挑战, 但随着该领域不断创新的理念和设备的出 
现，不远的未来流动化学在药物合成领域一定可以占有 一席之地。

\section{References}

[1] (a) Manz, A.; Graber, N.; Widmer, H. M. Sens. Actuators B 1990, $1,244$.

(b) http://pubs.rsc.org/en/journals/journalissues/lc.

(c) Haswell, S. J.; Middleton, R. J.; O’Sullivan, B.; Skelton, V.; Wattsa, P.; Styringb, P. Chem. Commun. 2001, 391.

(d) http://en.wikipedia.org/wiki/Flow chemistry.

(e) http://www.jflowchemistry.com.

(f) Elizabeth, F. New Synthetic Technologies in Medicinal Chemistry, RSC publishing, 2012

[2] Some selected books on Microreaction technology, see: (a) Ehrfeld W.; Hessel V.; Loewe, H. Microreactors: New Technology for Modern Chemistry, Wiley-VCH, Weinheim, 2000.

(b) Wirth, T. Microreactors in Organic Synthesis and Catalysis, Wiley-VCH, Weinheim, 2008.

(c) Yoshida, J.-I. Flash Chemistry: Fast Organic Synthesis in Microsystems, Wiley-VCH, Weinheim, 2008.

(d) Wiles, C.; Watts, P. Micro Reaction Technology in Organic Synthesis, CRC Press Inc., Boca Raton, 2011.

[3] Some selected international conferences on Microreaction technology, see: (a) International Conference on Microreaction Technology, 1997 2012.

(b) International Symposium on Micro Chemical Process and Synthesis, 2008.

(c) The 2nd Asia-Pacific Chemical and Biological Microfluidics Conference, 2011.

(d) The 3rd European Conference on Microfluidics, 2012.

[4] Some review articles of flow chemistry on pharmaceutical synthesis, see: (a) Kockmann, N.; Roberge, D. M. Chem. Eng. Technol. 2009, 32, 1682 .

(b) Malet-Sanz, L.; Susanne, F. J. Med. Chem. 2012, 55, 4062.

(c) Anderson, N. G. Org. Process Res. Dev. 2012, 16, 852.

[5] (a) Schwesinger, N.; Marufke, O.; Qiao, F.; Devant, R.; Wurziger, H. In Process Miniaturization: 2nd International Conference on Microreaction Technology, Eds: Ehrfeld, W.; Rinard, I. H.; Wegeng, R. S., AIChE, New Orleans, 1998, p. 124.

(b) Krummradt, H.; Kopp, U.; Stoldt, J. In Microreaction Technology: 3rd International Conference on Microreaction Technology, Ed.: Ehrfeld, W., Springer-Verlag, Berlin, 2000, p. 181.

[6] (a) Wörz, O.; Jäckel, K.-P.; Richter, T.; Wolf, A. Chem. Ing. Tech. 2000, 72, 460 .

(b) Wörz, O.; Jäckel, K.-P.; Richter, T.; Wolf, A. Chem. Eng. Technol. 2001, 24, 138.

[7] (a) Skelton, V.; Grenway, G. M.; Haswell, S. J.; Styring, P.; Morgan, D. O.; Warrington, B. H.; Wong, S. Y. F. Analyst 2001, 126, 11.

(b) Skelton, V.; Greenway, G. M.; Haswell, S. J.; Styring, P.; Morgan, D. O.; Warrington, B. H.; Wong, S. 4th International Conference on Microreaction Technology, Atlanta, USA, 2001, p. 78.

[8] (a) Wiles, C.; Watts, P.; Haswell, S. J.; Pombo-Villar, E. Lab Chip 2001, 1,100 .

(b) Watts, P.; Wiles, C.; Haswell, S. J.; Pombo-Villar, E. Lab Chip 2002, 2,141 .

(c) Wiles, C.; Watts, P.; Haswell, S. J.; Pombo-Villar, E. Lab Chip 2004, 4, 171.

[9] (a) Fernandez-Suarez, M.; Wong, S.Y. F.; Warrington, B. H. Lab on Chip 2002, 2,170. (b) Garcia-Egido, E.; Spikmans, V.; Wong, S. Y. F.; Warrington, B. H. Lab Chip 2003, 3, 67.

[10] (a) Yoshida, J.-I.; Okamoto, H. In Advanced Micro and Nanosystems Vol. 5. Micro Process Engineering, Ed.: Kockmann, N., WILEY-VCH, Weinheim, 2006.

(b) Kawaguchi, T.; Miyata, H.; Ataka, K.; Mae, K.; Yoshida, J.-I. Angew. Chem., Int. Ed. 2005, 44, 2413.

[11] (a) Bohn, L.; Braune, S.; Kotthaus, M.; Kraut, M.; Pöchlauer, P. Vorbach, M.; Wenka, A.; Schubert, K. 9th International Conference on Microreaction Technology, Potsdam, 2006.

(b) Ondrey, G. Chem. Eng. 2011, 118, 16.

[12] (a) Roberge, D. M.; Bieler, N.; Thalmann, M. PharmaChem 2006, 28,14 .

(b) Roberge, D. M. AIChE Spring National Meeting, Houston, TX, 2007, published on CD.

(c) Kockmann, N.; Gottsponer, M.; Zimmermann, B.; Roberge, D. M. Chem. Eur. J. 2008, 14, 7470.

[13] (a) Malet-Sanz, L.; Madrzak, J.; Holvey, R. S.; Underwood, T. Tetrahedron Lett.2009, 50, 7263.

(b) Malet-Sanz, L.; Madrzak, J.; Ley, S. V.; Baxendale, I. R. Org. Biomol. Chem. 2010, 8, 5324.

(c) Grafton, M.; Mansfield, A. C.; Fray, M. J. Tetrahedron Lett. 2010, 51, 1026.

[14] (a) Gutmann, B.; Roduit, J. P.; Roberge, D.; Kappe, C. O. Angew. Chem., Int. Ed. 2010, 49, 7101.

(b) Ye, X.; Johnson, M. D.; Diao, T.; Yates, M. H.; Stahl, S. S. Green Chem. 2010, 12, 1180.

[15] Pelleter, J.; Renaud, F. Org. Process Res. Dev. 2009, 13, 698.

[16] Abele, S.; Hö ck, S.; Schmidt, G.; Funel, J.-A.; Marti, R. Org. Process Res. Dev. 2012, 16, 1114.

[17] Some selected recent review articles on flow chemistry, see: (a) Wiles, C.; Watts, P. Chem. Commun. 2011, 47, 6512.

(b) Calabrese, G. S.; Pissavini, S. AIChE 2011, 57, 828.

(c) Hartman, R. L.; McMullen, J. P.; Jensen, K. F. Angew. Chem., Int. Ed. 2011, 50, 7502 .

(d) Wegner, J.; Ceylan, S.; Kirschning, A. Adv. Synth. Catal. 2012, $354,17$.

[18] Roberge, D. M.; Ducry, L.; Bieler, N.; Cretton, P.; Zimmermann, B. Chem. Eng. Technol. 2005, 28, 318.

[19] Some articles about continuous vs. batch, see: (a) Valera, F. E.; Quaranta, M.; Moran, A.; Blacker, J.; Armstrong, A.; Cabral, J. T.; Blackmond, D. G. Angew. Chem., Int. Ed. 2010, 49, 2478.

(b) Schaber, S. D.; Gerogiorgis, D. I.; Ramachandran, R.; Evans, J. M. B.; Barton, P. I.; Trout, B. L. Ind. Eng. Chem. Res. 2011, 50, 10083 .

Solid-involved flow process, see: (c) Kelly, C. B.; Lee, C. X.; Leadbeater, N. E. Tetrahedron Lett. 2011, 52, 263.

(d) Sedelmeier, J.; Ley, S. V.; Baxendale, I. R.; Baumann, M. Org Lett. 2010, 12, 3618 .

(e) Zhao, C.; He, L.; Qiao, S. Z.; Middelberg, A. P. J. Chem. Eng. Sci. 2011, 66, 1463.

(f) Hartman, R. L.; Naber, J. R.; Zaborenko, N.; Buchwald, S. L.; Jensen, K. F. Org. Process Res. Dev. 2010, 14, 1347.

[20] Lee, C.-C.; Sui, G.; Elizarov, A.; Shu, C. J.; Shin, Y.-S.; Dooley, A. N.; Huang, J.; Daridon, A.; Wyatt, P.; Stout, D.; Kolb, H. C.; Witte, O. N.; Satyamurthy, N.; Heath, J. R.; Phelps, M. E.; Quake, S. R.; Tseng, H.-R. Science 2005, 310, 1793.

[21] Baxendale, I. R.; Griffiths-Jones, C. M.; Ley, S. V.; Tranmer, G. F. Synlett 2006, 427.

[22] Zhang, X.; Stefanick, S.; Villani, F. J. Org. Process Res. Dev. 2004, 8, 455 .

[23] (a) Kim, H.; Nagaki, A.; Yoshida, J.-i. Nat. Commun. 2011, 2, 264. 
(b) Gustafsson, T.; Sö rensen, H.; Pontén, F. Org. Process Res. Dev. 2012, dx.doi.org/10.1021/op200340c.

[24] (a) Hogan, J. Nature 2006, 442, 351.

(b) Hübner, S.; Bentrup, U.; Budde, U.; Lovis, K.; Dietrich, T.; Freitag, A.; Küpper, L.; Jähnisch, K. Org. Process Res. Dev. 2009, $13,952$.

[25] (a) Jähnisch, K.; Dingerdissen, U. Chem. Eng. Technol. 2005, 28, 426.

(b) Lévesque, F.; Seeberger, P. H. Org. Lett. 2011, 13, 5008-5011.

[26] (a) Oelgemöller, M. Chem. Eng. Technol. 2012, 35, 1.

(b) Shinichiro, F.; Nobutake, T.; Yoshida, F.; Yoshida, H.; Doi, T. ; Takahashi, T. Chem. Commun. 2010, 46, 8722.

[27] (a) http://en.wikipedia.org/wiki/Lasker_Award.

(b) Dhainaut, J.; Dlubala, A.; Guevel, R.; Medard, A.; Oddon, G.; Raymond, N.; Turconi, J. WO 2011/026865, 2011 [Chem. Abstr. 2011, 154, 310801].

(c) Lévesque, F.; Seeberger, P. H. Angew. Chem., Int. Ed. 2012, 1706.

[28] (a) Kobayashi, J.; Mori, Y.; Okamoto, K.; Akiyama, R.; Ueno, M.; Kitamori, T.; Kobayashi, S. Science 2004, 304, 1305

(b) Jones, R. V.; Godorhazy, L.; Varga, N.; Szalay, D.; Urge, L.; Darvas, F. J. Comb. Chem. 2006, $8,110$.

(c) http://www.thalesnano.com/products/h-cube

[29] (a) Ley, S. V.; Schucht, O.; Thomas, A. W.; Murray, P. J. J. Chem. Soc., Perkin Trans. 1 1999, 1251.

(b) Baxendale, I. R.; Deeley, J.; Griffiths-Jones, C. M.; Ley, S. V.; Saaby, S.; Tranmer, G. K. Chem. Commun. 2006, 2566.

[30] (a) Capdeville, R.; Buchdunger, E.; Zimmermann, J.; Matter, A. Nat. Rev. Drug Discovery 2002, 1, 493.

(b) Arora, A.; Scholar, E. M. J. Pharmacol. Exp. Ther. 2005, 315, 971.

(c) Hopkin, M. D.; Baxendale, I. R.; Ley, S. V. Chem. Commun. 2010, 46, 2450.

[31] (a) Willging, E. M. Anal. Chem. 1987, 59, 938.

(b) Loupy, A. Microwaves in Organic Synthesis, John Wiley, New York, 2002, p. 61

[32] (a) Torborg, C.; Beller, M. Adv. Synth. Catal. 2009, 351, 3027.

(b) Glasnova, T. N.; Kappe, C. O. Adv. Synth. Catal. 2010, 352, 3089 .

(c) Gustafsson, T.; Pontn, F.; Seeberger, P. H. Chem. Commun. 2008, 1100 .

(d) Bedore, M. W.; Zaborenko, N.; Jensen, K. F.; Jamison, T. F. Org. Process Res. Dev. 2010, 14, 432.

(e) Glasnov, T. N.; Findenig, S.; Kappe, C. O. Chem. Eur. J. 2009, 15,1001 .

(f) Gutmann, B.; Roduit, J.-P.; Roberge, D.; Kappe, C. O. Angew. Chem., Int. Ed. 2010, 49, 7101.

[33] (a) Kim, E. G.; Schmidt, K.; Caseri, W. R.; Kreouzis, T.; Stingelin-Stutzmann, N.; Bredas, J. L. Adv. Mater. 2006, 18, 2039.

(b) Caseri, W. R.; Platinum Met. Rev. 2004, 48, 91.

(c) Pedrick, E. A.; Leadbeater, N. E. Inorg. Chem. Commun. 2011,
$14,481$.

[34] Bogdan, A. R.; Poe, S. L.; Kubis, D. C.; Broadwater, S. J.; McQuade, D. T. Angew. Chem., Int. Ed. 2009, 48, 8547.

[35] Baumann, M.; Baxendale, I. R.; Brasholz, M.; Hayward, J. J.; Ley, S. V.; Nikbin, N. Synlett 2011, 1375.

[36] Cervera-Padrell, A. E.; Nielsen, J. P.; Pedersen, M. J.; Christensen, K. M.; Mortensen, A. R.; Skovby, T.; Dam-Johansen, K.; Kiil, S.; Gernaey, K. V. Org. Process Res. Dev. 2012, 16, 901.

[37] (a) Moorhouse, A. D.; Moses, J. E. Chem. Soc. Rev. 2007, 36, 1249. (b) Baxendale, I. R.; Ley, S. V.; Mansfield, A. C.; Smith, C. D. Angew. Chem., Int. Ed. 2009, 48, 4017.

[38] (a) Daugan, A.; Grondin, P.; Ruault, C.; Le Monnier de Gouville, A.-C.; Coste, H.; Linget, J.-M.; Kirilovsky, J.; Hyafil, F.; Labaudinière, R. J. Med. Chem. 2003, 46, 4533.

(b) Mezencev, R.; Updegrove, T.; Kutschy, P.; Repovská, M.; McDonald, J. F. J. Nat. Med. 2011, 65, 488.

(c) Moody, C. J.; Roffey, J. R. A.; Swann, E.; Lockyer, S.; Houlbrook, S.; Stratford, I. J. Anti-Cancer Drugs 1999, 10, 577.

(d) Pagano, N; Heil, M. L.; Cosford, N. D. P. Synthesis 2012, 2537.

[39] Bonrath, W.; Karge, R.; Netscher, T. J. Mol. Catal. B: Enzym. 2002, $19 \sim 20,67$.

[40] Schwalbe, T.; Autze, V.; Wille, G. Chimia 2002, 56, 636.

[41] Choe, J.; Song, K.-H.; Kwon, Y. 4th Asia-Pacific Chemical Reaction Engineering Symposium, Gyeongju, Korea, 2005, p. 435.

[42] Sugimoto, A.; Sumino, Y.; Takagi, M.; Fukuyama, T.; Ryu, I. Tetrahedron Lett. 2006, 47, 6197.

[43] Pitts, M. R.; McCormack, P.; Whittall, J. Tetrahedron 2006, 62, 4705.

[44] Gustafsson, T.; Pontén, F.; Seeberger, P. H. Chem. Commun. 2008, 1100.

[45] LaPorte, T. L.; Hamedi, M.; DePue, J. S.; Shen, L.; Watson, D.; Hsieh, D. Org. Process Res. Dev. 2008, 12, 956.

[46] Grant, D.; Dahl, R.; Cosford, N. D. P. J. Org. Chem. 2008, 73, 7219.

[47] Kopach, M. E.; Murray, M. M.; Braden, T. M.; Kobierski, M. E.; Williams, O. L. Org. Process Res. Dev.2009, 13, 152.

[48] Buddoo, S.; Siyakatshana, N.; Zeelie, B.; Dudas, J. Chem. Eng. Process. 2009, 48, 1419.

[49] Tanaka, K.; Miyagawa, T.; Fukase, K. Synlett 2009, 1571.

[50] Tanaka, K.; Fukase, K. Org. Process Res. Dev.2009, 13, 983.

[51] Bogdan, A. R.; James, K. Chem. Eur. J. 2010, 16, 14506.

[52] Brasholz, M.; Johnson, B. A.; Macdonald, J. M.; Polyzos, A.; Tsanaktsidis, J.; Saubern, S.; Holmes, A. B.; Ryan, J. H. Tetrahedron 2010, 66, 6445.

[53] Qian, Z.; Baxendale, I. R.; Ley, S. V. Synlett 2010, 505.

[54] Brasholz, M.; Macdonald, J. M.; Saubern, S.; Ryan, J. H.; Holmes, A. B. Chem. Eur. J. 2010, 16, 11471.

[55] Qian, Z.; Baxendale, I. R.; Ley, S. V. Chem. Eur. J. 2010, 16, 12342.

[56] Noel, T.; Kuhn, S; Musacchio, A. J.; Jensen, K. F.; Buchwald, S. L. Angew. Chem., Int. Ed. 2011, 50, 5943. 